\title{
A Multi-Region Nonlinear Size-Structured Population Model with Coagulation and Vertical Effects
}

\section{Un modèle de population multirégional et non linéaire structuré par la taille avec coagulation et effets verticaux}

\author{
Azmy S. Ackleh ${ }^{1}$ and Robert L. Miller ${ }^{2, *}$ \\ ${ }^{1}$ Department of Mathematics \\ ackleh@louisiana.edu \\ ${ }^{2}$ Department of Civil Engineering, University of Louisiana at Lafayette, \\ Lafayette, LA 70504, USA \\ robert.miller@louisiana.edu
}

ABSTRACT. A general nonlinear model describing the evolution of size-structured populations influenced by coagulation and vertical effects is presented. The nonlinear population dynamics occur in a multi-region setting in which the transfer, coagulation, and vital rates of an individual depend on a vector describing conditions in the environment. The model for these dynamics consists of a system of two-dimensional nonlinear-nonlocal hyperbolic partial differential equations coupled with a system of one-dimensional nonlocal differential equations parametrized by a vertical location coordinate $z$ describing the environmental time-dynamics. A finite difference approximation approach is employed to study the wellposedness of the model and convergence of the scheme to the unique weak solution is established. Several examples are presented to illustrate the generality of the model and to motivate applications.

KEYWORDS. multi-region model, coagulation, vertical effects, difference approximations, convergence.

\section{Introduction}

Size-structured population models are encountered in many settings including spatial ecology, microbiology, as well as chemical and environmental engineering [17, 21, 26, 30, 31, 32, 40, 41]. The fate of a biological population of individuals often depends non-linearly upon many factors including resource competition which in turn affects the vital rates of individuals (e.g., growth, reproduction, and mortality). The population dynamics may also depend on other complex factors such as coagulation (e.g. flocculation of aggregates in microbial populations or school formation in fish) and fragmentation or breakage. Dispersal of individuals between spatial locations can also play a role in determining the fate of a given population particularly in natural environments (e.g., patch selection on a landscape, or vertical sinking and longitudinal drift of suspended particles due to currents).

For particular examples, the size-specific effects of physical coagulation (or aggregation) and biological cell growth have been suggested as important mechanisms shaping phytoplankton dynamics [25, 27, 37]. However size-structured models which account for coagulation have mostly been limited to a single region [4, 5, 6, 8]. Natural systems (e.g., lakes and oceans) feature hydrodynamic currents which introduce heterogeneity in the nutrient and temperature fields affecting phytoplankton growth. Eco-hydrodynamic system models can adequately account for spatialization effects via coupling between the hydrodynamics and ecological network models [19, 22]. But these typically do not resolve key microbiological processes such as growth and coagulation of individual phytoplankton particles [36, 42]. This limitation

\footnotetext{
${ }^{*}$ Corresponding author.
} 
is generally a consequence of the computational expense involved with resolving widely varying scales in time and space and a lack of size-specific field data on the populations [11, 23]. Faugeras and Maury present an explicit coupling of continuously structured population dynamics with a large-scale hydrodynamic system model to solve a parameter estimation problem involving skipjack tuna in the Indian Ocean [17]. Additional research is needed in the development of size-structured models capable of resolving important biological processes such as size-specific effects (e.g., growth and coagulation) as well as spatio-temporal dynamics (e.g., particle drift due to ocean currents or wind-driven seed dispersal).

From an analytical perspective, Ackleh and Ferdinand analyzed a size-structured model for a phytoplankton population with coagulation to simulate the effects of light shading on the population dynamics [2]. The model tracks the phytoplankton density at different depths in the water column and accounts for the effects of light extinction (hence the decline of photosynthesis) at greater depths. Therein, the existence-uniqueness of solutions to the quasilinear initial-boundary value problem was established via the method of finite differences. Ferdinand extended this work by considering vertical environmental effects in [20] and also demonstrated existence and uniqueness of weak solutions using the finite difference approach. The phytoplankton coagulation model coupled with a more general environment without vertical effects was analyzed in [7] and well-posedness was again established using the finite difference method. This work also featured a least-squares parameter estimation problem which was solved using the SIGMA tank experimental dataset. In [35], existence and uniqueness of a phytoplankton aggregation model which includes fragmentation was established using semigroup techniques in the absence of an explicit environmental coupling. Faugeras and Maury analyzed a generic multi-region model for an agesize structured fish population and established the existence and uniqueness of a positive weak solution using fixed point arguments [18].

In this paper we consider a general model inclusive of the nonlinear size-structured coagulation models analyzed in [2, 7, 20] in a multi-region setting. This general model will also take into account spatialization by allowing for transport of individuals in the population across multiple regions. The inclusion of these effects give rise to a highly nonlinear nonlocal system of partial differential equations (PDE's) describing the evolution of the population densities in each region coupled with a system of nonlocal parametrized differential equations which describe the environmental time-dynamics at various locations. This nonlinear PDE system notably features parameters which depend on vertically-varying nonlocal factors. It is well known that solutions of systems of scalar conservation laws may become discontinuous so we seek weak solutions in the space of bounded variation $B V$ functions. Our goal is to establish existence and uniqueness of weak solutions of the general nonlinear model using a finite difference approach as in [2, 7]. In particular, we develop a semi-implicit finite difference approximation for the model, establish a priori estimates for this approximation and apply a standard compactness argument used in conservation laws [39] to show the convergence of the approximation to the unique weak solution. This approach also provides a convergent and stable numerical scheme to simulate the model dynamics.

This paper is organized as follows: we introduce the model in Section 2 and in Section 3 we introduce the notation and assumptions used in the forthcoming analysis. In Sections 4 and 5 we establish a series of technical lemmas which establish important stability properties of the finite difference scheme. In Section 6 we show using a standard compactness argument that the finite difference approximations converge to the unique weak solution of the model. Finally, motivating applications and numerical experiments are furnished in Section 7 and topics for future research are discussed in Section 8 , 


\section{The Model}

Consider a network of sub-regions consisting of size-structured populations in which vertical effects may be present. Individuals can be transported between the regions based on various factors in a general way. Examples may include fish or phytoplankton aggregates being passively transported by water currents, seeds being carried by the wind, or populations migrating to find resources. Motivated by existing models of phytoplankton aggregate populations and other types of biological systems which prominently feature aggregation and sinking effects, the model also features a coagulation process. We also take into account spatial variability along a vertical coordinate direction. From hereinafter we take $x_{\max }, z_{\max }$ and $T$ as fixed positive real numbers. The following model describes the coupled system $p=1, \ldots, n \in \mathbb{N}$ and $\ell=1, \ldots, m \in \mathbb{N}$ :

$$
\begin{aligned}
& \left(u_{p}\right)_{t}+\left(g_{p} u_{p}\right)_{x}+\left(w_{p} u_{p}\right)_{z}+\mu_{p} u_{p}=S_{p}^{B} \\
& \quad+\sum_{i=1}^{n} Q_{p, i} u_{i}-\sum_{i=1}^{n} Q_{i, p} u_{p}+\mathcal{A}_{p}\left(u_{p}\right), \quad(t, x, z) \in(0, T] \times\left(0, x_{\text {max }}\right] \times\left(0, z_{\max }\right] \\
& \left.\left(g_{p} u_{p}\right)\right|_{x=0}=F_{p}(t, z, \vec{Y})+\sum_{i=1}^{n} \int_{0}^{x_{\max }} \beta_{p, i}(t, x, z, \vec{\phi}, \vec{Y}) u_{i}(t, x, z) d x \\
& \left.\left(w_{p} u_{p}\right)\right|_{z=0}=\alpha_{p}(t, x, \vec{\Phi}), \quad u_{p}(0, x, z)=u_{p}^{0}(x, z) \\
& \left(Y_{p, \ell}\right)_{t}=\sum_{i=1}^{n} \mathcal{Q}_{p, i, \ell} Y_{i, \ell}-\sum_{i=1}^{n} \mathcal{Q}_{i, p, \ell} Y_{p, \ell}+f_{p, \ell}-h_{p, \ell} Y_{p, \ell}, \quad Y_{p, \ell}(0, z)=Y_{p, \ell}^{0}(z) .
\end{aligned}
$$

Here $u_{p}=u_{p}(t, x, z)$ is the population density of particle aggregates in region $p$ having size $x$ at a location $z$ from a fixed reference at time $t$. For example, such a datum can be taken as the surface of the ocean or a lake in the particular case of phytoplankton dynamics. The individual growth rate, $g_{p}=g_{p}(t, x, z, \vec{\phi}, \vec{Y})$ depends on the size of the individual, distance from the datum, time, and the effects of biological competition $\vec{\phi}$ and the environment $\vec{Y}$ occurring in the various regions. For example, $\vec{Y}$ may track the evolution of nutrients, zooplankton, or the population dynamics of other species affecting the food web. The term $w_{p}=w_{p}(t, x, z, \vec{\phi}, \vec{Y})$ is the settling velocity of an aggregate which also varies depending on size, vertical location, time, competition, and the environment. Similarly, the term $\mu_{p}=\mu_{p}(t, x, z, \vec{\phi}, \vec{Y})$ represents the individual aggregate mortality rate. The term $S_{p}^{B}=S_{p}^{B}(t, x, z, \vec{\phi}, \vec{Y})$ represents the addition of aggregates from an external (exogenous) source, and the term $Q_{i, p}=Q_{i, p}(t, x, z, \vec{\phi}, \vec{Y})$ measures the endogenous transfer rate of aggregates from region $p$ into region $i$ at location $z$ from the datum. The coagulation functional $\mathcal{A}_{p}=\mathcal{A}_{p}\left(u_{p}\right)$ assumes the following form:

$$
\begin{array}{r}
\mathcal{A}_{p}\left(u_{p}\right)=\frac{1}{2} \int_{0}^{x} \eta_{p}(t, x-s, s, z, \vec{\phi}, \vec{Y}) u_{p}(t, x-s, z) u_{p}(t, s, z) d s \\
-\int_{0}^{x \max } \eta_{p}(t, x, s, z, \vec{\phi}, \vec{Y}) u_{p}(t, x, z) u_{p}(t, s, z) d s
\end{array}
$$

where the function $\eta_{p}$ is the rate at which an aggregate of volume $x$ inside of region $p$ adheres to an aggregate of volume $s$ when they coalesce at location $z$. The first term in $\mathcal{A}_{p}\left(u_{p}\right)$ depicts the rate of formation of particles due to binary collisions of smaller sized particles whose combined volumes add up to $x$. The second term describes the rate at which such collisions cause these particles to be lost from the same interval. The parameter $x_{\max }$ represents the volume of the largest sized particle in the study. The vector $\vec{\phi}=\left(\phi_{1}, \ldots, \phi_{n}\right)^{\mathrm{T}}$, where $\mathrm{T}$ denotes the transpose of a vector, represents a weight of the population where the weighted population at time $t$ and location $z$ in region $p$ is given by:

$$
\phi_{p}(t, z)=\int_{0}^{z} \int_{0}^{x_{\max }} \theta_{p}(x, \zeta) u_{p}(t, x, \zeta) d x d \zeta, \quad p=1, \ldots, n .
$$

For convenience of notation we define the total weighted average in region $p$ at time $t$ as $\Phi_{p}(t) \equiv$ $\phi_{p}\left(t, z_{\max }\right)$ with weight $\theta_{p}$. 
The nonlocal boundary condition at $x=0$ includes the function $F_{p}(t, z, \vec{Y})$ representing inflow of size zero individuals entering into region $p$ from an external source. The boundary condition also includes the effects of reproduction which are modeled by the second term where $\beta_{p, i}$ is the birth rate of particles from region $i$ into region $p$ for all $i, p=1, \ldots, n$. The term $\alpha_{p}(t, x, \vec{\Phi})$ represents the influx of individuals of size $x$ at time $t$ at the datum, and the function $u_{0}(x, z)$ is the initial population density.

The dynamics of the environment $\vec{Y}$ are assumed to vary with time $t$ and vertical location $z$. We also assume that the settling velocities of the $\vec{Y}$ variables are negligible. As such the environment is modeled with a system of nonlocal parametrized differential equations where $Y_{p, \ell}=Y_{p, \ell}(t, z)$ is the environmental variable $\ell$ in region $p$ at time $t$ and location $z$ for $\ell=1, \ldots, m$ and $p=1, \ldots, n$. The term $\mathcal{Q}_{i, p, \ell}=\mathcal{Q}_{i, p, \ell}(t, z, \vec{\sigma}, \vec{Y})$ measures the endogenous transfer rate of the environmental variable $\ell$ from region $p$ into region $i$ at vertical location $z$ and the sums reflect the combined effects of endogenous transfer. The terms $f_{p, \ell}=f_{p, \ell}(t, z, \vec{\sigma}, \vec{Y})$ and $h_{p, \ell}=h_{p, \ell}(t, z, \vec{\sigma}, \vec{Y})$ represent source and mortality terms respectively. The components of the functional $\vec{\sigma}$ are given by

$$
\sigma_{p, \ell}(t, z)=\int_{0}^{z} \int_{0}^{x_{\max }} \gamma_{p, \ell}(x, \zeta) u_{p}(t, x, \zeta) d x d \zeta,
$$

where $\gamma_{p, \ell}$ is a weight function. The term $\sigma_{p, \ell}$ represents weighted averages of the total population with respect to the weight $\gamma_{p, \ell}$ in compartment $p$ for environmental variable $\ell$ at time $t$ and location $z$. This term may be used to represent, e.g., the effects of biological competition on resource consumption where the time-varying resources are represented by components of $\vec{Y}$.

It is worth pointing out that the model parameters depend on the total environment $(\vec{Y}=$ $\left.\left[\left[Y_{1,1}, \ldots, Y_{1, m}\right]^{\mathrm{T}}, \ldots,\left[Y_{n, 1}, \ldots, Y_{n, m}\right]^{\mathrm{T}}\right]\right)$ and overall measures of competition factors $\left(\vec{\phi}=\left[\phi_{1}, \ldots, \phi_{n}\right]^{\mathrm{T}}\right.$, $\left.\vec{\Phi}=\left[\Phi_{1}, \ldots, \Phi_{n}\right]^{\mathrm{T}}\right)$. This flexibility is motivated by the potential need for dynamic adjustment of forcing conditions (e.g., dilution/flow rates, seeding frequency) in various applications.

\section{Assumptions and Notation}

The space of continuous functions over $\Omega \subset \mathbb{R}^{d}, d \in \mathbb{N}$ is denoted by $C(\Omega)$. The symbol $C^{1}(\Omega)$ is used to denote the space of continuously differentiable functions on $\Omega$, and we use $B V(\Omega)$ to denote the space of functions with bounded total variation on $\Omega$. The function $h: \Omega \rightarrow \mathbb{R}$ is said to be locally Lipschitz continuous if for every $x \in \Omega$ there exists a neighborhood $U$ of $x$ such that $h$ restricted to $U$ is Lipschitz. The symbol $\operatorname{Lip}_{l o c}(\Omega)$ denotes the space of locally Lipschitz continuous functions on $\Omega$ and $\operatorname{Lip}(\Omega)$ denotes the space of Lipschitz continuous functions on $\Omega$. Throughout the paper we will use the shorthand notation $L_{h_{\ell}}$ to represent the Lipschitz constant for a Lipchitz continuous function $h_{\ell}$. We omit the index $\ell$ when denoting the maximal Lipschitz constant for a given function over all indices, i.e., $L_{\gamma} \equiv \max \left\{L_{\gamma_{1}}, \cdots, L_{\gamma_{m}}\right\}$.

Let $c_{0}, c_{1}$, be sufficiently large positive constants. Denote by $\mathbb{R}_{+}^{d}$ the positive cone of $\mathbb{R}^{d}, d \in \mathbb{N}$ and let $\mathbb{D}=[0, T] \times\left[0, x_{\text {max }}\right] \times\left[0, x_{\text {max }}\right] \times\left[0, z_{\text {max }}\right] \times \mathbb{R}_{+}^{n(m+1)}, \mathbb{D}_{1} \equiv[0, T] \times\left[0, x_{\text {max }}\right] \times\left[0, z_{\text {max }}\right] \times \mathbb{R}_{+}^{n(m+1)}$, $\mathbb{D}_{2}=[0, T] \times\left[0, x_{\text {max }}\right] \times \mathbb{R}_{+}^{n(m+1)}, \mathbb{D}_{3}=[0, T] \times\left[0, z_{\text {max }}\right] \times \mathbb{R}_{+}^{n(m+1)}, \mathbb{D}_{4}=[0, T] \times\left[0, z_{\text {max }}\right] \times \mathbb{R}_{+}^{n m}$, $|\vec{\phi}|_{1}=\sum_{p=1}^{n}\left|\phi_{p}\right|$, and $|\vec{Y}|_{\infty}=\sum_{p=1}^{n} \sum_{\ell=1}^{m} \max _{z \in\left[0, z_{\max }\right]}\left|Y_{p, \ell}\right|$. Throughout the discussion we impose the following conditions on our model parameters in (2.1), for each $\ell=1,2, \ldots, m$ and $i, p=1, \ldots, n$. 
(B1) The functions $g_{p}, w_{p} \in C^{1}\left(\mathbb{D}_{1}\right)$ are nonnegative, and $\left(g_{p}\right)_{x},\left(g_{p}\right)_{z},\left(w_{p}\right)_{x},\left(w_{p}\right)_{z} \in \operatorname{Lip}_{\overrightarrow{\text { loc }}}\left(\mathbb{D}_{1}\right)$. Moreover, there exist constants $\zeta_{1}, \zeta_{2}>0$ such that $g_{p}(t, 0, z, \vec{\phi}, \vec{Y}) \geq \zeta_{1}>0, w_{p}(t, x, 0, \vec{\phi}, \vec{Y}) \geq \zeta_{2}>0$.

(B2) $Q_{i, p}, \mu_{p}, S_{p}^{B} \in \operatorname{Lip}_{\mathrm{loc}}\left(\mathbb{D}_{1}\right)$ are nonnegative. Furthermore, $Q_{i, p} \leq c_{0}$ and for each $t \geq 0, S_{p}^{B} \leq$ $c_{1}+c_{1}|\vec{\phi}|_{1}+c_{1}|\vec{Y}|_{\infty}$

(B3) $\mathcal{Q}_{i, p, \ell}, f_{p, \ell}, h_{p, \ell} \in \operatorname{Lip}_{\text {loc }}\left(\mathbb{D}_{3}\right)$ are nonnegative, $\mathcal{Q}_{i, p, \ell} \leq c_{0}$, and for each $t \geq 0$ we have $f_{p, \ell}, h_{p, \ell} \leq$ $c_{1}+c_{1}|\vec{\sigma}|_{1}+c_{1}|\vec{Y}|_{\infty}$.

(B4) $\beta_{i, p} \in \operatorname{Lip}_{\mathrm{loc}}\left(\mathbb{D}_{1}\right)$ is nonnegative and $\beta_{i, p} \leq c_{0}$.

(B5) $F_{p} \in \operatorname{Lip}_{\text {loc }}\left(\mathbb{D}_{4}\right)$ and $\alpha_{p} \in \operatorname{Lip}_{\text {loc }}\left(\mathbb{D}_{2}\right)$ are nonnegative, $F_{p} \leq c_{1}+c_{1}|\vec{Y}|_{\infty}$, and $\alpha_{p} \leq c_{0}$.

(B6) The weight functions $\theta_{p}, \gamma_{p, \ell} \in \operatorname{Lip}\left(\mathbb{R}^{2}\right)$ are nonnegative and $\theta_{p}+\gamma_{p, \ell} \leq c_{0}$.

(B7) $u_{p}^{0}$, and $Y_{p, \ell}^{0}$ are nonnegative, $u_{p}^{0} \in B V\left(\left[0, x_{\max }\right] \times\left[0, z_{\max }\right]\right)$, and $Y_{p, \ell}^{0} \in \operatorname{Lip}\left[0, z_{\max }\right]$.

(B8) $\eta_{p} \in \operatorname{Lip}_{\text {loc }}(\mathbb{D})$, is symmetric, i.e., $\eta_{p}(t, x, s, z, \vec{\phi}, \vec{Y})=\eta_{p}(t, s, x, z, \vec{\phi}, \vec{Y})$, nonnegative, and $\eta_{p}(t, x, s, z, \vec{\phi}, \vec{Y}) \leq c_{0}$. Furthermore, $\eta_{p}(t, x, s, z, \vec{\phi}, \vec{Y})=0$ if $x+s>x_{\text {max }}$ and $\eta_{p}(t, x, 0, z, \vec{\phi}, \vec{Y})$ $=0$.

We define the strip over which the variables $u_{p}$ and $Y_{p, \ell}$ are defined

$$
\mathbb{D}_{x} \equiv[0, T] \times\left[0, x_{\max }\right] \times\left[0, z_{\max }\right], \quad \mathbb{D}_{z} \equiv[0, T] \times\left[0, z_{\max }\right],
$$

for all $p=1, \ldots, n$, and $\ell=1 \ldots, m$ and next define a weak solution for (2.1).

Definition 3.1. An $n(m+1)$-tuple $(\vec{u}, \vec{Y}) \in \Pi_{p=1}^{n} L^{\infty}\left(\mathbb{D}_{x}\right) \times \Pi_{\ell=1}^{m} \Pi_{p=1}^{n} L^{\infty}\left(\mathbb{D}_{z}\right)$ is called a weak solution to problem (2.1) if for every positive integer $p=1, \ldots, n$, and for each $t \in(0, T], u_{p}(t, \cdot, \cdot) \in$ $B V\left(\left[0, x_{\max }\right] \times\left[0, z_{\max }\right]\right)$, and also satisfies

$$
\begin{aligned}
& \int_{0}^{z_{\max }} \int_{0}^{x_{\max }}\left(\rho_{p}(t, x, z) u_{p}(t, x, z)-\rho_{p}(0, x, z) u_{p}(0, x, z)\right) d x d z-\int_{0}^{t} \int_{0}^{z_{\max }} \int_{0}^{x_{\max }} \frac{\partial \rho_{p}}{\partial t} u_{p} d x d z d t \\
& +\int_{0}^{t} \int_{0}^{z_{\max }} \rho_{p}\left(t, x_{\max }, z\right) g_{p}\left(t, x_{\max }, z, \vec{\phi}, \vec{Y}\right) u_{p}\left(t, x_{\max }, z\right) d z d t \\
& -\int_{0}^{t} \int_{0}^{z_{\max }}\left(\rho_{p} F_{p}+\rho_{p} \sum_{i=1}^{n} \int_{0}^{x_{\max }} \beta_{p, i} u_{i} d x\right) d z d t-\int_{0}^{t} \int_{0}^{z_{\max }} \int_{0}^{x_{\max }} \frac{\partial \rho_{p}}{\partial x} g_{p} u_{p} d x d z d t \\
& +\int_{0}^{t} \int_{0}^{x_{\max }}\left(\rho_{p}\left(t, x, z_{\max }\right) w_{p}\left(t, x, z_{\max }\right) u_{p}\left(t, x, z_{\max }\right)-\rho_{p}(t, x, 0) \alpha_{p}(t, x, \vec{\Phi})\right) d x d t \\
& -\int_{0}^{t} \int_{0}^{z_{\max }} \int_{0}^{x_{\max }} \frac{\partial \rho_{p}}{\partial z} w_{p} u_{p} d x d z d t+\int_{0}^{t} \int_{0}^{z_{\max }} \int_{0}^{x_{\max }} \rho_{p} \mu_{p} u_{p} d x d z d t \\
& +\int_{0}^{t} \int_{0}^{z_{\max }} \int_{0}^{x_{\max }} \rho_{p} \sum_{i=1}^{n} Q_{i, p} u_{p} d x d z d t \\
& =\frac{1}{2} \int_{0}^{t} \int_{0}^{z_{\max }} \int_{0}^{x_{\max }} \int_{0}^{x} \rho_{p}(t, x, z) \eta_{p}(t, x-s, s, z, \vec{\phi}, \vec{Y}) u_{p}(t, x-s, z) u_{p}(t, s, z) d s d x d z d t \\
& -\int_{0}^{t} \int_{0}^{z_{\max }} \int_{0}^{x_{\max }} \rho_{p} u_{p}(t, x, z) \int_{0}^{x_{\max }} \eta_{p}(t, x, s, z, \vec{\phi}, \vec{Y}) u_{p}(t, s, z) d y d x d z d t \\
& +\int_{0}^{t} \int_{0}^{z_{\max }} \int_{0}^{x_{\max }} \rho_{p} S_{p}^{B} d x d z d t+\int_{0}^{t} \int_{0}^{z_{\max }} \int_{0}^{x_{\max }} \rho_{p} \sum_{i=1}^{n} Q_{p, i} u_{i} d x d z d t,
\end{aligned}
$$

for every test function $\rho_{p} \in C^{1}\left((0, T) \times\left(0, x_{\max }\right) \times\left(0, z_{\max }\right)\right)$ and for each $z \in\left(0, z_{\max }\right] Y_{p, \ell}(\cdot, z) \in$ $C[0, T]$ and also satisfies

$$
\begin{aligned}
Y_{p, \ell}(t, z)= & Y_{p, \ell}(0, z)+\sum_{i=1}^{n} \int_{0}^{t} \mathcal{Q}_{p, i, \ell}(s, z, \vec{\sigma}, \vec{Y}) Y_{i, \ell}(s, z) d s-\sum_{i=1}^{n} \int_{0}^{t} \mathcal{Q}_{i, p, \ell}(s, z, \vec{\sigma}, \vec{Y}) Y_{p, \ell}(s, z) d s \\
& +\int_{0}^{t}\left[f_{p, \ell}(s, z, \vec{\sigma}, \vec{Y})-h_{p, \ell}(s, z, \vec{\sigma}, \vec{Y}) Y_{p, \ell}(s, z)\right] d s,
\end{aligned}
$$

for all $p=1,2, \ldots, n$ and $\ell=1,2, \ldots, m$. 


\section{Finite Difference Approximations}

Suppose that the intervals $[0, T],\left[0, x_{\max }\right]$ and $\left[0, z_{\max }\right]$ are divided into $L, N$ and $R$ subintervals, respectively. The following notation will be used throughout this paper: $\Delta t=T / L, \Delta x=x_{\max } / N$ and $\Delta z=z_{\max } / R$ denote the time and spatial mesh sizes respectively. The mesh points are given by: $t^{k}=k \Delta t, k=0,1,2, \ldots, L, x_{j}=j \Delta x, j=0,1,2, \ldots, N$ and $z_{l}=l \Delta z, l=0,1,2, \ldots, R$. For convenience of notation we apply equal mesh sizes within a given region $p$ and a uniform time step. The proof of convergence can be established in a similar manner using a more general non-uniform mesh. We denote by $u_{p, j, l}^{k}, Y_{p, \ell, l}^{k}, \phi_{p, l}^{k}$, and $\sigma_{p, \ell, l}^{k}$, the finite difference approximations of $u_{p}\left(t^{k}, x_{j}, z_{l}\right), Y_{p, \ell}\left(t^{k}, z_{l}\right)$, $\phi_{p}\left(t^{k}, z_{l}\right)$, and $\sigma_{p, \ell}\left(t^{k}, z_{l}\right)$ respectively. The values of the model parameters evaluated at the mesh points are given below for all $i, p=1, \ldots, n, j, s=0, \ldots, N, l=0, \ldots, R, k=0, \ldots, L$, and $\ell=1, \ldots, m$,

$$
\begin{aligned}
& \beta_{p, i, j, l}^{k}=\beta_{p, i}\left(t^{k}, x_{j}, z_{l}, \vec{\phi}^{k}, \vec{Y}^{k}\right), g_{p, j, l}^{k}=g_{p}\left(t^{k}, x_{j}, z_{l}, \vec{\phi}^{k}, \vec{Y}^{k}\right), \mu_{p, j, l}^{k}=\mu_{p}\left(t^{k}, x_{j}, z_{l}, \vec{\phi}^{k}, \vec{Y}^{k}\right), \\
& S_{p, j, l}^{B,}=S_{p}^{B}\left(t^{k}, x_{j}, z_{l}, \vec{\phi}^{k}, \vec{Y}^{k}\right), F_{p, l}^{k}=F_{p}\left(t^{k}, z_{l}, \vec{Y}^{k}\right), Q_{p, i, j, l}^{k}=Q_{p, i}\left(t^{k}, x_{j}, z_{l}, \vec{\phi}^{k}, \vec{Y}^{k}\right), \\
& f_{p, \ell, l}^{k}=f_{p, \ell}\left(t^{k}, z_{l}, \vec{\sigma}^{k}, \vec{Y}^{k}\right), h_{p, \ell, l}^{k}=h_{p, \ell}\left(t^{k}, z_{l}, \vec{\sigma}^{k}, \vec{Y}^{k}\right), \eta_{p, j, s, l}^{k}=\eta_{p}\left(t^{k}, x_{j}, x_{s}, z_{l}, \vec{\phi}^{k}, \vec{Y}^{k}\right), \\
& \theta_{p, j, l}=\theta_{p}\left(x_{j}, z_{l}\right), w_{p, j, l}^{k}=w_{p}\left(x_{j}, z_{l}, t^{k}, \vec{Y}^{k}\right), \gamma_{p, \ell, j, l}=\gamma_{p, \ell}\left(x_{j}, z_{l}\right), \mathcal{Q}_{p, i, l}^{k}=\mathcal{Q}_{p, i}\left(t^{k}, z_{l}, \vec{\sigma}^{k}, \vec{Y}^{k}\right), \\
& \phi_{p, l}^{k}=\sum_{q=1}^{l} \sum_{j=1}^{N} \theta_{p, j, q} u_{p, j, q}^{k} \Delta x \Delta z, \sigma_{p, \ell, l}^{k}=\sum_{q=1}^{l} \sum_{j=1}^{N} \gamma_{p, \ell, j, q} u_{p, j, q}^{k} \Delta x \Delta z, \alpha_{p, j}^{k}=\alpha_{p}\left(t^{k}, x_{j}, \vec{\Phi}^{k}\right)
\end{aligned}
$$

and $\Phi_{p}^{k}=\phi_{p, R}^{k}$. We will use the following notation and define the $\ell^{1}, \ell^{\infty}$, norms and the $T V$ (total variation) seminorm of the grid functions $u_{p, j, l}^{k}$ and $\vec{Y}^{k}$ by

$$
\begin{aligned}
& \left\|u_{p}^{k}\right\|_{1}=\sum_{l=1}^{R} \sum_{j=1}^{N}\left|u_{p, j, l}^{k}\right| \Delta x \Delta z, \quad\left\|\vec{u}^{k}\right\|_{1}=\sum_{p=1}^{n}\left\|u_{p}^{k}\right\|_{1}, \quad\left\|u_{p}^{k}\right\|_{\infty}=\max _{0 \leq j \leq N ; 0 \leq l \leq R}\left|u_{p, j, l}^{k}\right|, \\
& \left\|\vec{u}^{k}\right\|_{\infty}=\sum_{p=1}^{n}\left\|u_{p}^{k}\right\|_{\infty}, \quad\left\|Y_{p, \ell}^{k}\right\|_{\infty}=\max _{0 \leq l \leq R}\left|Y_{p, \ell, l}^{k}\right|, \quad\left\|\vec{Y}^{k}\right\|_{\infty}=\sum_{p=1}^{n} \sum_{\ell=1}^{m}\left\|Y_{p, \ell}^{k}\right\|_{\infty} \\
& T V_{x}\left(u_{p}^{k}\right)=\sum_{l=1}^{R} \sum_{j=0}^{N-1}\left|u_{p, j+1, l}^{k}-u_{p, j, l}^{k}\right| \Delta z, \quad T V_{z}\left(u_{p}^{k}\right)=\sum_{j=1}^{N} \sum_{l=0}^{R-1}\left|u_{p, j, l+1}^{k+1}-u_{p, j, l}^{k+1}\right| \Delta x \\
& T V\left(u_{p}^{k}\right)=T V_{x}\left(u_{p}^{k}\right)+T V_{z}\left(u_{p}^{k}\right), \quad T V_{x}\left(\vec{u}^{k}\right)=\sum_{p=1}^{n} T V_{x}\left(u_{p}^{k}\right), \quad T V_{z}\left(\vec{u}^{k}\right)=\sum_{p=1}^{n} T V_{z}\left(u_{p}^{k}\right), \\
& T V\left(\vec{u}^{k}\right)=T V_{x}\left(\vec{u}^{k}\right)+T V_{z}\left(\vec{u}^{k}\right)=\sum_{p=1}^{n} T V\left(u_{p}^{k}\right)
\end{aligned}
$$

for $k=0,1, \ldots, L$ and $p, i=1,2, \ldots, n$. We omit the subscripts when denoting the uniform upper bound over all the regions, i.e., $\|\eta\|_{\infty}=\max _{p=1, \ldots, n}\left\|\eta_{p}\right\|_{\infty}$. We also adopt a first-order nonstandard finite difference scheme valid for all $p=1, \ldots, n, \ell=1, \ldots, m, j=1, \ldots, N, l=1, \ldots, R$ :

$$
\begin{aligned}
& \quad \frac{u_{p, j, l}^{k+1}-u_{p, j, l}^{k}}{\Delta t}+\frac{g_{p, j, l}^{k} u_{p, j, l}^{k+1}-g_{p, j-1, l}^{k} u_{p, j-1, l}^{k+1}}{\Delta x}+\frac{w_{p, j, l}^{k} u_{p, j, l}^{k+1}-w_{p, j, l-1}^{k} u_{p, j, l-1}^{k+1}}{\Delta z} \\
& \quad+\mu_{p, j, l}^{k} u_{p, j, l}^{k+1}+\sum_{i=1}^{n} Q_{i, p, j, l}^{k} u_{p, j, l}^{k+1}+u_{p, j, l}^{k+1} \sum_{q=1}^{N} \eta_{p, q, j, l}^{k} u_{p, q, l}^{k} \Delta x \\
& =\frac{1}{2} \sum_{s=1}^{j-1} \eta_{p, j-s, s, l}^{k} u_{p, j-s, l}^{k} u_{p, s, l}^{k+1} \Delta x+S_{p, j, l}^{B+k}+\sum_{i=1}^{n} Q_{p, i, j, l}^{k} u_{i, j, l}^{k} \\
& g_{p, 0, l}^{k} u_{p, 0, l}^{k+1}=F_{p, l}^{k}+\sum_{i=1}^{n} \sum_{j=1}^{N} \beta_{p, i, j, l}^{k} u_{i, j, l}^{k} \Delta x, w_{p, j, 0}^{k} u_{p, j, 0}^{k+1}=\alpha_{p, j}^{k}, u_{p, j, l}^{0}=u_{p}^{0}\left(x_{j}, z_{l}\right) \\
& \quad \frac{Y_{p, \ell, l}^{k+1}-Y_{p, \ell, l}^{k}}{\Delta t}=\sum_{i=1}^{n} \mathcal{Q}_{p, i, \ell, l}^{k} Y_{i, \ell, l}^{k}-\sum_{i=1}^{n} \mathcal{Q}_{i, p, \ell, l}^{k} Y_{p, \ell, l}^{k+1}+f_{p, \ell, l}^{k}-h_{p, \ell, l}^{k} Y_{p, \ell, l}^{k+1}, Y_{p, \ell, l}^{0}=Y_{p, \ell}^{0}\left(z_{l}\right) .
\end{aligned}
$$

First observe that the first equation in (4.1) has a unique nonnegative algebraic solution and this result satisfies a biological requirement. To establish this, for convenience of notation let

$$
\mathbb{I}_{p, j, l}^{k+1}=\frac{\Delta t}{2} \sum_{s=1}^{j-1} \eta_{p, j-s, s, l}^{k} u_{p, j-s, l}^{k} u_{p, s, l}^{k+1} \Delta x+\Delta t S_{p, j, l}^{B, k}+\Delta t \sum_{i=1}^{n} Q_{p, i, j, l}^{k} u_{i, j, l}^{k}
$$

and

$$
\mathbb{J}_{p, j, l}^{k}=\Delta t \sum_{i=1}^{n} Q_{i, p, j, l}^{k}+\Delta t \sum_{q=1}^{N} \eta_{p, q, j, l}^{k} u_{p, q, l}^{k} \Delta x+\Delta t \mu_{p, j, l}^{k} .
$$

noting that both $\mathbb{I}_{p, j, l}^{k+1}$ and $\mathbb{J}_{p, j, l}^{k}$ are nonnegative assuming that the initial and boundary conditions are nonnegative. Next multiplying the first row of (4.1) by $\Delta t$ and rewriting the equation we obtain

$$
u_{p, j, l}^{k+1}=\frac{u_{p, j, l}^{k}+\frac{\Delta t}{\Delta x} g_{p, j-1, l}^{k} u_{p, j-1, l}^{k+1}+\frac{\Delta t}{\Delta z} w_{p, j, l-1}^{k} u_{p, j, l-1}^{k+1}+\mathbb{I}_{p, j, l}^{k+1}}{1+\frac{\Delta t}{\Delta x} g_{p, j, l}^{k}+\frac{\Delta t}{\Delta z} w_{p, j, l}^{k}+\mathbb{J}_{p, j, l}^{k}}
$$


All terms on the right hand side of (4.2) are nonnegative by the nonnegativity assumption (B8) on the initial data $u^{0}$. Furthermore, the solution to (4.2) is obtained first by applying the surface and reproductive boundary conditions respectively and then applying a left-to-right substitution process proceeding in a downward manner. From assumption (B3), and (B8), the algebraic solution to the system of differential equations given by the third equation in (4.1) is also nonnegative since this equation can be rewritten as

$$
Y_{p, \ell, l}^{k+1}=\frac{Y_{p, \ell, l}^{k}+f_{p, \ell, l}^{k} \Delta t+\sum_{i=1}^{n} \mathcal{Q}_{p, i, \ell, l}^{k} Y_{i, \ell, l}^{k} \Delta t}{1+\sum_{i=1}^{n} \mathcal{Q}_{i, p, \ell, l}^{k} \Delta t+h_{p, \ell, l}^{k} \Delta t},
$$

and the condition $Y_{p, \ell}^{0} \equiv Y_{p, \ell}(0) \geq 0$ immediately establishes $Y_{p, \ell, l}^{k+1} \geq 0$ for all $k=0, \ldots, L-1$ by assumption (B3). This establishes nonnegativity of the numerical scheme (4.1).

\section{Estimates for the Finite Difference Approximations}

We begin with the following lemma, the proof of which is similar to that of Lemma 4.1 in [7].

Lemma 5.1. Assume that $u_{p, j, l}^{k}$ is nonnegative. Then for any $N \geq 1$ we have the following:

$\frac{1}{2} \sum_{j=1}^{N} \sum_{s=1}^{j-1} \eta_{p, j-s, s, l}^{k} u_{p, j-s, l}^{k} u_{p, s, l}^{k+1}-\sum_{j=1}^{N} u_{p, j, l}^{k+1} \sum_{q=1}^{N} \eta_{p, q, j, l}^{k} u_{p, q, l}^{k}=-\frac{1}{2} \sum_{j=1}^{N} u_{p, j, l}^{k+1} \sum_{q=1}^{N} \eta_{p, q, j, l}^{k} u_{p, q, l}^{k}$.

Next we establish an $\ell_{1}$ bound on the numerical approximations.

Lemma 5.2. The scheme is bounded in $\ell_{1}$, i.e., there exists a constant $B_{1}$ such that for all $l=0, \ldots, R$

$$
\left\|\vec{u}^{k+1}\right\|_{1}+\left\|\vec{Y}^{k+1}\right\|_{\infty} \leq B_{1}
$$

Proof. See appendix.

Observe that by nonnegativity of the scheme and Lemma 5.2 we have for $l=1, \ldots, R$,

$$
\phi_{p, l}^{k+1} \leq \phi_{p, R}^{k+1}=\sum_{q=1}^{R} \sum_{j=1}^{N} \theta_{p, j, q} u_{p, j, q}^{k+1} \Delta x \Delta z \leq\|\theta\|_{\infty}\left\|\vec{u}^{k+1}\right\|_{1} \leq c_{0} B_{1} \leq B_{1,1},
$$

and similarly $\sigma_{p, \ell} \leq B_{1,1}$ for some sufficiently large constant $B_{1,1}$. The boundedness of $S_{p}^{B}, f_{p, \ell}, h_{p, \ell}$, and $F_{p}$ (by $B_{1,1}$ without loss of generality) also follow directly from the bounds on the functional terms, the fact that $\vec{Y}^{k}$ is bounded (by Lemma 5.2), and assumptions (B2), (B3), and (B6). Thus, using (B1)(B8), we obtain a uniform upper bound $B_{1,1}$ on the parameters $g_{p}, w_{p}, \mu_{p}$ over the compact set $[0, T] \times$ $\left[0, x_{\max }\right] \times\left[0, z_{\max }\right] \times \Pi_{s=1}^{n}\left[0, B_{1,1}\right] \times \Pi_{s=1}^{n m}\left[0, B_{1}\right]$ and their first partial derivatives. We also note that the first partial derivatives of $\eta_{p}, \beta_{i, p}$ are also bounded over a compact set and for convenience we denote this bound by $B_{1,1}$ as well. The existence of the bound $B_{1,1}$ also establishes that for any sufficiently small $\delta_{1}, \delta_{2}>0$ there exists a constant $\lambda$ such that

$$
\begin{aligned}
\max _{p=1, \ldots, n}\left\{\operatorname { s u p } _ { ( x , z , t , \vec { \phi } , \vec { Y } ) \in \mathbb { D } } \left\{\mu_{p}(t, x, z, \vec{\phi}, \vec{Y})\right.\right. & +\left|\frac{g_{p}\left(t, x+\delta_{1}, z, \vec{\phi}, \vec{Y}\right)-g_{p}(t, x, z, \vec{\phi}, \vec{Y})}{\delta_{1}}\right| \\
+ & \left.\left.\left|\frac{w_{p}\left(t, x, z+\delta_{2}, \vec{\phi}, \vec{Y}\right)-w_{p}(t, x, z, \vec{\phi}, \vec{Y})}{\delta_{2}}\right|\right\}\right\} \leq \lambda .
\end{aligned}
$$

The preceding estimate $(5.1)$ is necessary to establish the following $\ell^{\infty}$ bound on the approximations. 
Lemma 5.3. The scheme is bounded in $\ell^{\infty}$, i.e., there exists a constant $B_{2}$ such that if $\Delta t$ satisfies $\max \left\{0.5 c_{0} B_{1}, \lambda\right\} \Delta t<1$,

$$
\left\|\vec{u}^{k+1}\right\|_{\infty} \leq B_{2}
$$

\section{Proof. See appendix.}

Lemma 5.4. There exist positive constants $B_{3, i}, i=1, \ldots, 8$, such that the following are satisfied:

(5.4.1) For all $p=1, \ldots, n ; j=1, \ldots, N ; l=1, \ldots, R$; and $k=0, \ldots, L-1$,

$$
\begin{aligned}
& \sum_{p=1}^{n}\left|\phi_{p, l}^{k}-\phi_{p, l-1}^{k}\right| \leq B_{3,1} \Delta z, \sum_{p=1}^{n} \sum_{\ell=1}^{m}\left|\sigma_{p, \ell, l}^{k}-\sigma_{p, \ell, l-1}^{k}\right| \leq B_{3,1} \Delta z \\
& \sum_{p=1}^{n} \sum_{\ell=1}^{m}\left|Y_{p, \ell, l}^{k}-Y_{p, \ell, l-1}^{k}\right| \leq B_{3,1} \Delta z
\end{aligned}
$$

(5.4.2) For all $p=1, \ldots, n ; j, s=1, \ldots, N ; l=1, \ldots, R$; and $k=0, \ldots, L-1$,

$$
\begin{aligned}
& \left|F_{p, l}^{k}-F_{p, l-1}^{k}\right| \leq B_{3,2} \Delta z,\left|\mu_{p, j, l}^{k}-\mu_{p, j, l-1}^{k}\right| \leq B_{3,2} \Delta z,\left|S_{p, j, l}^{B, k}-S_{p, j, l-1}^{B, k}\right| \leq B_{3,2} \Delta z \\
& \left|Q_{p, i, j, l}^{k}-Q_{p, i, j, l-1}^{k}\right| \leq B_{3,2} \Delta z,\left|\eta_{p, j, s, l}^{k}-\eta_{p, j, s, l-1}^{k}\right| \leq B_{3,2} \Delta z \\
& g_{p, j, l}^{k}-g_{p, j, l-1}^{k}\left|\leq B_{3,2} \Delta z,\right| \beta_{p, i, j, l}^{k}-\beta_{p, i, j, l-1}^{k} \mid \leq B_{3,2} \Delta z .
\end{aligned}
$$

(5.4.3) For all $p=1, \ldots, n ; j=0, \ldots, N-1 ; l=1, \ldots, R$; and $k=0, \ldots, L-1$,

$$
\begin{aligned}
& \left|\alpha_{p, j+1}^{k}-\alpha_{p, j}^{k}\right| \leq B_{3,3} \Delta x,\left|g_{p, j+1, l}^{k}-g_{p, j, l}^{k}\right| \leq B_{3,3} \Delta x,\left|\beta_{p, i, j+1, l}^{k}-g_{p, i, j, l}^{k}\right| \leq B_{3,3} \Delta x \\
& \left|\mu_{p, j+1, l}^{k}-\mu_{p, j, l}^{k}\right| \leq B_{3,3} \Delta x,\left|S_{p, j+1, l}^{B, k}-S_{p, j, l}^{B, k}\right| \leq B_{3,3} \Delta x \\
& \left|Q_{p, i, j+1, l}^{k}-Q_{p, i, j, l}^{k}\right| \leq B_{3,3} \Delta x,\left|\eta_{p, j+1, s, l}^{k}-\eta_{p, j, s, l}^{k}\right| \leq B_{3,3} \Delta x .
\end{aligned}
$$

(5.4.4) For all $p=1, \ldots, n ; j=1, \ldots, N ; l=1, \ldots, R$; and $k=0, \ldots, L-1$,

$$
\left|\left(g_{p, j, l}^{k}-g_{p, j-1, l}^{k}\right)-\left(g_{p, j, l-1}^{k}-g_{p, j-1, l-1}^{k}\right)\right| \leq B_{3,4} \Delta x \Delta z .
$$

(5.4.5) For all $p=1, \ldots, n ; j=1, \ldots, N ; l=1, \ldots, R$; and $k=0, \ldots, L-1$,

$$
\left|\left(w_{p, j, l}^{k}-w_{p, j-1, l}^{k}\right)-\left(w_{p, j, l-1}^{k}-w_{p, j-1, l-1}^{k}\right)\right| \leq B_{3,5} \Delta x \Delta z .
$$

(5.4.6) For all $i, p=1, \ldots, n ; j=1, \ldots, N ; l=0, \ldots, R$; and $k=0, \ldots, L-1$,

$$
\begin{aligned}
& \sum_{p=1}^{n}\left|\phi_{p, l}^{k+1}-\phi_{p, l}^{k}\right| \leq B_{3,6} \Delta t, \sum_{p=1}^{n} \sum_{\ell=1}^{m}\left|\sigma_{p, \ell, l}^{k+1}-\sigma_{p, \ell, l}^{k}\right| \leq B_{3,6} \Delta t \\
& \sum_{p=1}^{n} \sum_{\ell=1}^{m}\left|\mathcal{Q}_{i, p, \ell, l}^{k+1}-\mathcal{Q}_{i, p, \ell, l}^{k}\right| \leq B_{3,6} \Delta t, \sum_{p=1}^{n} \sum_{\ell=1}^{m}\left|Y_{p, \ell, l}^{k+1}-Y_{p, \ell, l}^{k}\right| \leq B_{3,6} \Delta t
\end{aligned}
$$

(5.4.7) For all $j, s=1, \ldots, N ; l=1, \ldots, R ; i, p=1, \ldots, n ; \ell=1, \ldots, m$; and $k=0, \ldots, L-1$,

$$
\begin{aligned}
& \left|\begin{array}{l}
g_{p, j, l}^{k+1}-g_{p, j, l}^{k}\left|\leq B_{3,7} \Delta t,\right| Q_{p, i, j, l}^{k+1}-Q_{p, i, j, l}^{k}\left|\leq B_{3,7} \Delta t,\right| \mu_{p, j, l}^{k+1}-\mu_{p, j, l}^{k} \mid \leq B_{3,7} \Delta t, \\
\eta_{p, j, s, l}^{k+1}-\eta_{p, j, s, l}^{k} \\
S_{p, j, l}^{B, k+1}-S_{p, j, l}^{B, k}
\end{array}\right| \leq B_{3,7} \Delta t,\left|\beta_{p, i, j, l}^{k+1}-\beta_{p, i, j, l}^{k}\right| \leq B_{3,7} \Delta t,\left|F_{p, l}^{k+1}-F_{p, l}^{k}\right| \leq B_{3,7} \Delta t,\left|\alpha_{p, j}^{k+1}-\alpha_{p, j}^{k}\right| \leq B_{3,7} \Delta t .
\end{aligned}
$$


(5.4.8) For all $p=1, \ldots, n$ and $k=0, \ldots, L-1$,

$$
\begin{aligned}
& \sum_{l=1}^{R-1} \frac{\Delta t}{\Delta x}\left|u_{p, 0, l+1}^{k+1}-u_{p, 0, l}^{k+1}\right| g_{p, 0, l}^{k} \Delta x \leq B_{3,8}\left(1+T V_{z}\left(\vec{u}^{k}\right)\right) \Delta t \\
& \sum_{j=1}^{N-1} \frac{\Delta t}{\Delta z}\left|u_{p, j+1,0}^{k+1}-u_{p, j, 0}^{k+1}\right| w_{p, j, 0}^{k} \Delta z \leq B_{3,8} \Delta t \\
& \sum_{l=1}^{R}\left(1-B_{3,2} \Delta t+\frac{\Delta t}{\Delta x} g_{p, 0, l}^{k}\right)\left|u_{p, 1, l}^{k+1}-u_{p, 0, l}^{k+1}\right| \Delta z \leq \sum_{l=1}^{R}\left|u_{p, 1, l}^{k}-u_{p, 0, l}^{k}\right| \Delta z \\
& +B_{3,8}\left(1+T V_{x}\left(\vec{u}^{k}\right)+T V_{z}\left(\vec{u}^{k}\right)\right) \Delta t, \\
& \sum_{j=1}^{N}\left(1-B_{3,3} \Delta t+\frac{\Delta t}{\Delta z} w_{p, j, 0}^{k}\right)\left|u_{p, j, 1}^{k+1}-u_{p, j, 0}^{k+1}\right| \Delta x \leq \sum_{j=1}^{N}\left|u_{p, j, 1}^{k}-u_{p, j, 0}^{k}\right| \Delta x+B_{3,8} \Delta t \text {. }
\end{aligned}
$$

Proof. See appendix.

Towards establishing the next Lemma let $B_{5,1}, B_{5,2}$ be constants satisfying

$$
\begin{aligned}
B_{5,1} \geq & \max \left\{0.5\|\eta\|_{\infty} B_{2} B_{1} x_{\max } z_{\max }+0.5 B_{3} B_{1} x_{\max } z_{\max }\right. \\
& \left.+B_{3} x_{\max } z_{\max }+(n+4) B_{3} B_{1}+B_{1}^{2} B_{3}, 0.5\|\eta\|_{\infty} B_{2} x_{\max },\|Q\|_{\infty}, B_{3}\right\} \\
B_{5,2} \geq & 2 \max \left\{1.5 B_{3} B_{2} B_{1} x_{\max }+B_{3} x_{\max } z_{\max }\right. \\
& \left.+(n+2) B_{3} B_{1}+2 B_{1}, 1.5 B_{2}\|\eta\|_{\infty} x_{\max },\|Q\|_{\infty}, B_{3}\right\} .
\end{aligned}
$$

Lemma 5.5. If $\Delta t$ satisfies $\left(2 B_{5,1}+B_{5,2}+B_{3,2}+B_{3,3}+B_{3,8}+\lambda\right) \Delta t<1$, there exists a constant $B_{5}$ such that for every nonnegative integer $k$,

$$
T V\left(\vec{u}^{k+1}\right) \leq B_{5}
$$

Proof. See appendix.

We now show that the approximations $u_{p, j, l}^{k}$ satisfy a Lipschitz condition in $t$ and that the approximations $Y_{p, \ell, l}^{k}$ satisfy a Lipschitz conditions in $t$ and $z$.

Lemma 5.6. There exists an $B_{6}>0$, independent of $\Delta x, \Delta t$, and $\Delta z$ such that

$$
\begin{aligned}
& \sum_{p=1}^{n} \sum_{l=1}^{R} \sum_{j=1}^{N}\left|\frac{u_{p, j, l}^{r}-u_{p, j, l}^{q}}{\Delta t}\right| \Delta x \Delta z \leq B_{6}|r-q|, \\
& \sum_{p=1}^{n} \sum_{\ell=1}^{m}\left|\frac{Y_{p, \ell, l}^{r}-Y_{p, \ell, l}^{q}}{\Delta t}\right| \leq B_{6}|r-q|, \quad \sum_{p=1}^{n} \sum_{\ell=1}^{m}\left|\frac{Y_{p, \ell, r}^{k}-Y_{p, \ell, q}^{k}}{\Delta z}\right| \leq B_{6}|r-q|
\end{aligned}
$$

Proof. See appendix.

Next we have the following lemma which guarantees the continuous dependence of the solutions $\left\{\vec{u}^{k}\right\}$ and $\left\{\vec{Y}^{k}\right\}$ to the coupled system (2.1) with respect to the initial conditions $\vec{u}^{0}, \vec{Y}^{0}$.

Lemma 5.7. Assume that $C_{6,1} \Delta t<1$ where $C_{6,1}=\max \left\{c_{0}, 1.5 c_{0} B_{2} x_{\max }\right\}$. Let $\left\{\vec{u}^{k}\right\},\left\{\overrightarrow{\hat{u}}^{k}\right\},\left\{\vec{Y}^{k}\right\}$ and $\left\{\overrightarrow{\hat{Y}}^{k}\right\}, k=0, \ldots, L-1$, be the solutions of (2.1) corresponding to the initial conditions $\vec{u}^{0}, \overrightarrow{\hat{u}}^{0}, \vec{Y}^{0}$, and $\overrightarrow{\hat{Y}}^{0}$, respectively. Then there exists a constant $C^{\dagger}>0$ such that

$$
\begin{aligned}
& \sum_{p=1}^{n}\left\|u_{p}^{k+1}-\hat{u}_{p}^{k+1}\right\|_{1}+\max _{l=0, \ldots, R} \sum_{p=1}^{n} \sum_{\ell=1}^{m}\left|Y_{p, \ell, l}^{k+1}-\hat{Y}_{p, \ell, l}^{k+1}\right| \\
& \quad \leq\left(\frac{1+C^{\dagger} \Delta t}{1-C_{6,1} \Delta t}\right)\left(\sum_{p=1}^{n}\left\|u_{p}^{k}-\hat{u}_{p}^{k}\right\|_{1}+\max _{l=0, \ldots, R} \sum_{p=1}^{n} \sum_{\ell=1}^{m}\left|Y_{p, \ell, l}^{k}-\hat{Y}_{p, \ell, l}^{k}\right|\right) .
\end{aligned}
$$

Proof. See appendix. 


\section{Existence and Uniqueness of Weak Solutions}

Following [39] (Chapter 16, Pgs. 275-279) we define two families of functions $\left\{U_{p, \Delta t, \Delta x, \Delta z}(t, x, z)\right\}$ for $(t, x, z) \in \mathbb{D}_{x}$ and $\left\{\mathcal{Y}_{p, \ell, \Delta t, \Delta z}(t, z)\right\}$ for $(t, z) \in \mathbb{D}_{z}$ by extending the approximations $u_{p, j, l}^{k}$ to step functions and $Y_{p, \ell, l}^{k}$ in (4.1) to piecewise linear functions in $t$ as follows:

$$
\begin{aligned}
& U_{p, \Delta t, \Delta x, \Delta z}(t, x, z)=u_{p, j, l}^{k} \text { for }(t, x, z) \in\left[t^{k-1}, t^{k}\right) \times\left[x_{j-1}, x_{j}\right) \times\left[z_{l-1}, z_{l}\right), \\
& \mathcal{Y}_{p, \ell, \Delta t, \Delta z}(t, z)=Y_{p, \ell, l}^{k-1}+\frac{Y_{p, \ell, l}^{k}-Y_{p, \ell, l}^{k-1}}{\Delta t}\left(t-t^{k-1}\right), \quad \text { for } \quad t \in\left[t^{k-1}, t^{k}\right) \times\left[z_{l-1}, z_{l}\right),
\end{aligned}
$$

for all $k=1,2, \ldots, L, j=1, \ldots, N, l=1, \ldots, R$. Then, utilizing the a-priori estimates established in the previous section and applying standard compactness arguments (see [15, 39]), one can show the following:

Theorem 6.1. For $p=1, \ldots, n$, and $\ell=1, \ldots, m$, there exists a subsequence $\left\{U_{p, \Delta t_{i}, \Delta x_{i}, \Delta z_{i}}\right\} \subset$ $\left\{U_{p, \Delta t, \Delta x, \Delta z}\right\}$ which converges to a function of bounded total variation $u_{p} \in L_{\infty}\left(\mathbb{D}_{x}\right)$, and a subsequence $\left\{\mathcal{Y}_{p, \ell, \Delta t_{j}, \Delta z_{j}}\right\} \subset\left\{\mathcal{Y}_{p, \ell, \Delta t, \Delta z}\right\}$ which converges to a function $Y_{p, \ell} \in{ }^{\infty}\left(\mathbb{D}_{z}\right)$ in the sense that for all $t>0$,

$$
\begin{aligned}
& \int_{0}^{z_{\max }} \int_{0}^{x_{\max }}\left|U_{p, \Delta t_{i}, \Delta x_{i}, \Delta z_{i}}(t, x, z)-u_{p}(t, x, z)\right| d x d z \rightarrow 0, \\
& \int_{0}^{T} \int_{0}^{z_{\max }} \int_{0}^{x_{\max }}\left|U_{p, \Delta x_{i}, \Delta z_{i}, \Delta t_{i}}(t, x, z)-u_{p}(t, x, z)\right| d x d z d t \rightarrow 0,
\end{aligned}
$$

and

$$
\sup _{t \in[0, T], z \in\left[0, z_{\text {max }}\right]}\left|\mathcal{Y}_{p, \ell, \Delta t_{j}, \Delta z_{j}}(t, z)-Y_{p, \ell}(t, z)\right| \rightarrow 0,
$$

as $i, j \rightarrow \infty$.

The next theorem proves that the limit functions constitute a weak solution of (2.1). The proof of this result uses similar arguments as in [7, 39].

Theorem 6.2. The limit functions $\vec{u}$ and $\vec{Y}$ constructed by the finite difference scheme in Theorem (6.1) define a weak solution of the model (2.1) and satisfy

$$
\begin{aligned}
& \left\|u_{p}\right\|_{L^{\infty}\left([0, T] \times\left[0, x_{\max }\right] \times\left[0, z_{\max }\right]\right)} \leq B_{1}, \\
& \left\|Y_{p, \ell}\right\|_{L^{\infty}\left([0, T] \times\left[0, z_{\max }\right]\right)} \leq B_{2},
\end{aligned}
$$

for each $t \in[0, T]$, for all $p=1, \ldots, n, \ell=1, \ldots, m$.

Now, using an approach similar to [9, 10], which relies on a continuous dependence estimate similar to the one in Lemma 5.7, we can establish the following result which shows that the weak solution in Theorem 6.1 and 6.2 is unique. Hence, we can lift the sub-sequential convergence of the finite difference scheme in Theorem 6.1 to convergence along the whole sequence to the unique weak solution.

Theorem 6.3. Suppose $\vec{u}, \vec{Y}$ and $\overrightarrow{\hat{u}}, \overrightarrow{\hat{Y}}$ are weak solutions of (2.1) corresponding to the initial conditions $\vec{u}^{0}, \vec{Y}^{0}$ and $\overrightarrow{\hat{u}}^{0}, \overrightarrow{\hat{Y}}_{p}^{0}$ respectively. Then there exists a constant $B_{7}$ such that for any $t>0$,

$$
\begin{aligned}
& \sum_{p=1}^{n}\left\|u_{p}(t, \cdot, \cdot)-\hat{u}_{p}(t, \cdot, \cdot)\right\|_{1}+\sum_{p=1}^{n} \sum_{\ell=1}^{m}\left\|Y_{p, \ell}(t, \cdot)-\hat{Y}_{p, \ell}(t, \cdot)\right\|_{\infty} \\
& \leq B_{7}\left(\sum_{p=1}^{n}\left\|u_{p}(0, \cdot, \cdot)-\hat{u}_{p}(0, \cdot, \cdot)\right\|_{1}+\sum_{p=1}^{n} \sum_{\ell=1}^{m}\left\|Y_{p, \ell}(0, \cdot)-\hat{Y}_{p, \ell}(0, \cdot)\right\|_{\infty}\right) .
\end{aligned}
$$




\section{Applications}

In this section we will present special cases of the general model that are of interest as well as some numerical simulations.

\subsection{Coupled system of $N$ size-structured populations}

The nonlinear model developed in [3] describes the dynamics of a coupled system featuring $N$ sizestructured populations. The subpopulations compete for common resources and may represent different physiological groups of the same species. The model is described by the following system:

$$
\begin{array}{ll}
\left(u_{i}\right)_{t}+\left(g_{i}(x, P) u_{i}\right)_{x}+m_{i}(x, P) u_{i}=0 & (t, x) \in(0, T] \times\left(0, x_{\max }\right], \\
g_{i}(t, 0) u_{i}(t, 0)=C_{i}(t)+\sum_{j=1}^{n} \int_{0}^{x_{\max }} \gamma_{i, j} \beta^{j}(x, P) u^{j}(t, x) d x & t \in(0, T] \\
u_{i}(0, x)=u_{i}^{0}(x) & x \in\left[0, x_{\max }\right]
\end{array}
$$

where $u_{i}(t, x)(i=1, \ldots, n)$ represents the population density of individuals belonging to the $i$-th subpopulation of size $x$ at time $t$ and

$$
P(t)=\sum_{j=1}^{n} \int_{0}^{x_{\max }} \hat{\theta}_{j}(x) u_{j}(t, x) d x
$$

The vital rate terms $g_{i}$ and $m_{i}$ are the individual growth and mortality rates, and $\beta^{j}$ is the fecundity rate of an individual in subpopulation $j$ having size $x$ at population level $P$. The term $\gamma_{i, j}$ measures the probability that an individual in subpopulation $j$ will reproduce an individual in subpopulation $i$. The term $C_{i}=C_{i}(t)$ is the inflow rate of the $i$-th subpopulation of smallest size individuals from external sources. In addition to analyzing competition effects between different ecotypes in very general settings, (7.1) could represent, e.g., the dynamics of competing species of size-structured phytoplankton in a well-mixed environment with abundant resources where coagulation effects are not considered. The model parameters and variables are related to the general model (2.1) in the following manner:

$$
\begin{gathered}
\beta_{i, j}=\gamma_{i, j} \beta^{j}, \quad w_{i}=0, \quad \mu_{i}=m_{i}, \quad \eta_{i}=0, \quad F_{i}=C_{i} \\
\alpha_{p}=0, \quad \theta_{i}=\hat{\theta}_{i}, \quad \phi_{i}=\int_{0}^{x_{\max }} \hat{\theta}_{i}(x) u_{i}(t, x) d x .
\end{gathered}
$$

Here we note that the effects of the environment $\vec{Y}$ are not considered and $F_{i}=F_{i}(t)$.

\subsection{Size-structured phytoplankton/nutrient dynamics: the single region case}

The model developed in [20] is used to investigate the dynamics of a phytoplankton cell population under the influence of coagulation and size-specific competition for nutrients. The model is described by the following system:

$$
\begin{aligned}
& \frac{\partial u}{\partial t}+(g(x, P(t, z), N(t, z)) u)_{x}+w(x) u_{z}=\mathcal{A}(u) \\
& g(0, P, N) u(t, 0, z)=\int_{0}^{x_{\max }} \beta(x, P, N) u(t, x, z) d x \\
& u(t, x, 0)=\alpha(t, x), \quad u(0, x, z)=u^{0}(x, z) \\
& \frac{\partial N}{\partial t}=\mathcal{F}(z, P, N), \quad N(0, z)=N^{0}(z) .
\end{aligned}
$$

where

$$
\begin{array}{r}
\mathcal{A}(u)=\frac{1}{2} \int_{0}^{x} \eta(x-s, s, z) u(t, x-s, z) u(t, s, z) d s \\
-u(t, x, z) \int_{0}^{x_{\max }} \eta(x, s, z) u(t, s, z) d s
\end{array}
$$


and

$$
P(t, z)=\int_{0}^{z} \int_{0}^{x_{\max }} x u(t, x, \zeta) d x d \zeta
$$

where $u=u(t, x, z)$ represents the density of phytoplankton aggregates of volume $x$ present at time $t$ and depth $z$ below the surface layer. The parameter $g(x, P, N)$ is the rate at which an aggregate of volume $x$ grows to the next larger size while $\beta(x, P, N)$ is the reproduction rate of a size $x$ aggregate or the rate at which single cells of phytoplankton break off aggregates having size $x$ during cell division. The function $w(x)$ represents the fall velocity of an aggregate of volume $x$ and $\alpha(t, x)$ is the density of aggregates of size $x$ at time $t$ in the surface layer. The function $u^{0}$ is the initial condition denoting aggregate density at time $t=0$. In the term $\mathcal{A}(u)$ described above, $\eta(x, y, z)$ is the coagulation kernel which is the rate at which an aggregate of size $x$ joins with an aggregate of size $y$ upon colliding together at depth $z$. The first term in $\mathcal{A}(u)$ describes the formation of aggregates in the size interval $[x, x+d x)$ owing to these collisions in the ocean while the second term describes the loss of aggregates from this size interval to larger size intervals. The variables and parameters are related to the general model as follows:

$$
\begin{aligned}
& n=1, \quad u_{1}=u, \quad g_{1}=g, \quad \mu_{1}=0, \quad \mathcal{A}_{1}=\mathcal{A}, \\
& F_{1}=0, \quad \beta_{1,1}=\beta, \quad \alpha_{1}=\alpha, \quad \theta_{1}=x, \quad \phi_{1}=P, \\
& m=1, \quad Y_{1}=N, \quad f_{1}=\mathcal{F}, \quad \eta_{1}=\eta, \quad \sigma_{1,1}=P .
\end{aligned}
$$

\subsection{Numerical examples}

Here, we develop test problems to demonstrate the order of convergence of the numerical method and to provide simple illustrations of the type of dynamics which can be analyzed with the model.

\subsubsection{Order of convergence of the numerical method}

The first trial was conducted to demonstrate the order of convergence of the numerical scheme. The model domain was taken as $x_{\max }=1, z_{\max }=1$, and $T=0.5$ with two regions $(p=1,2)$. For the sake of simplicity we assume that $\eta_{p}=\beta_{p}=0$ for the convergence trial and take the growth and settling rates as fixed constants $g_{p}=w_{p}=1(p=1,2)$. The mortality rates are given by $\mu_{1}=0, \mu_{2}=2$ and the flow rates are given by $Q_{2,1}=1$ and $Q_{1,2}=0$. The environment effects $\vec{Y}$ and endogenous sources $S_{p}^{B}(p=1,2)$ were neglected for this example. For convenience we set $F_{p}=\exp (t-z)$ and $\alpha_{p}=\exp (t-x)$ for the zero size and depth boundaries respectively $(p=1,2)$. The initial conditions were given by $u_{p}(x, z)=\exp (-x-z)$. The preceding choice of rate parameters, forcing terms, and initial conditions give rise to the following variant of (2.1):

$$
\begin{aligned}
& \left(u_{1}\right)_{t}+\left(u_{1}\right)_{x}+\left(u_{1}\right)_{z}=-Q_{2,1} u_{1}, \quad(t, x, z) \in(0,0.5] \times(0,1] \times(0,1] \\
& \left(u_{2}\right)_{t}+\left(u_{2}\right)_{x}+\left(u_{2}\right)_{z}+2 u_{2}=Q_{2,1} u_{1}, \quad(t, x, z) \in(0,0.5] \times(0,1] \times(0,1] \\
& \left(g_{p} u_{p}\right)(t, 0, z)=\exp (t-z) \quad\left(w_{p} u_{p}\right)(t, x, 0)=\exp (t-x), \quad p=1,2 .
\end{aligned}
$$

One can easily verify that $u_{p}(t, x, z)=\exp (t-x-z)(p=1,2)$ solves (7.3). We analyzed convergence of the method (4.1) by using the scheme to numerically solve (7.3) and comparing the simulation results against the exact solution. The $\ell^{1}$ errors and order of convergence are defined in the following manner:

$$
E_{1}^{\Delta x, \Delta z}=\sum_{p=1}^{2} \sum_{j=1}^{N_{1}} \sum_{l=1}^{N_{2}}\left|u_{p}\left(t^{L}, x_{j}, z_{l}\right)-u_{p, j, l}^{L}\right| \Delta x \Delta z ; \quad O_{\ell^{1}}=\log _{2}\left(\frac{E_{1}^{\Delta x, \Delta z}}{E_{1}^{\Delta x / 2, \Delta z / 2}}\right) .
$$


We used $\Delta x=1 / N, \Delta z=1 / R$ and $\Delta t=\Delta x / 16$ to compute solutions at various resolutions $N=R=$ $2,4,8,16,32,64,128$ and $L=16,32,64,128,256,512,1024$ respectively, and the errors were analyzed at $T=0.5$. Table 1 suggests that the numerical method achieves first order convergence.

Tableau 1. Numerical convergence summary: $\ell^{1}$ errors and order of convergence.

\begin{tabular}{cccccc}
\hline$N_{1}$ & $N_{2}$ & $N_{1} \times N_{2}$ & $L$ & $E_{1}^{\Delta x, \Delta z}$ & $O_{\ell^{1}}$ \\
\hline 2 & 2 & 4 & 16 & 0.0785604 & - \\
4 & 4 & 16 & 32 & 0.0481935 & 0.7049625 \\
8 & 8 & 64 & 64 & 0.0269130 & 0.8405362 \\
16 & 16 & 256 & 128 & 0.0144168 & 0.9005564 \\
32 & 32 & 1,024 & 256 & 0.0076038 & 0.9229449 \\
64 & 64 & 4,096 & 512 & 0.0039073 & 0.9605650 \\
128 & 128 & 16,384 & 1,024 & 0.0019806 & 0.9802040 \\
\hline
\end{tabular}

\subsubsection{An example from limnology and oceanography}

This final trial was performed to demonstrate utility of the model and numerical method in a practical setting. To do this we perform simulations to investigate the role played by physical mechanisms on key phenomena observed in the study of phytoplankton populations in lakes and oceans. We focus here on the vertical distribution of the biomass in a hypothetical phytoplankton population subject to water currents. It is well known that natural phytoplankon populations exhibit chlorophyll maximums below the free surface (termed deep chlorophyll maximums - DCM's), and these maximums correlate positively with biomass levels [28]. The vertical position of these peaks is also attributed to gradients in light availability and nutrients - both of which depend generally on ambient concentrations of phytoplankton. The topic of DCM's is a very rich area of research and questions about the variability of the DCM are a central aspect of these studies [16].

In this exercise we analyze how DCM's may respond to physical mechanisms. The effects we analyze here are vertical velocity/flow profiles acting in tandem with particle sinking effects. For this simple example we presume that the effects of vertical advection and turbulent vertical diffusion are negligible. We also assume a homogeneous environment with abundant resources so as to isolate the effects. We apply the model (2.1) to a situation involving the evolution of a size-structured population density $u_{p}(t, x, y, z)$ moving through four (4) spatial regions $(p=1, \ldots, 4)$ subject to the physical effects of coagulation and sinking. An example of such a situation occurs in the case of suspended phytoplankton particles in a stratified hydrodynamic system (e.g., low-gradient river, lake or ocean). We take the convention that positive flow occurs in the direction of increasing $p$, i.e., $Q_{p}>0$ implies that the flow $Q_{p}$ carries particle aggregates into region $p+1$ and although the model (2.1) allows bi-directional flow, to simplify the forthcoming analysis we assume flow only occurs in one direction. The population density starts from an initial concentration in the first region $u_{1}^{0}$ which is distributed uniformly across all size classes $x$ and depths $z$. For convenience, the model domain was taken as $x_{\max }=1, z_{\max }=1000$, and $T=1$. Although we do not specify exact units in this simple example, typical units for $x$ and $z$ in real-world phytoplankton systems are $\mathrm{mm}^{3}$ and meters respectively with $T$ typically on the order of days [7]. 
$(\mathrm{C}-1)$

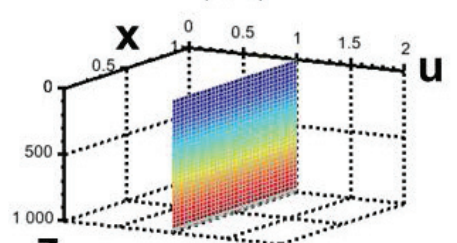

$\mathbf{Z}$

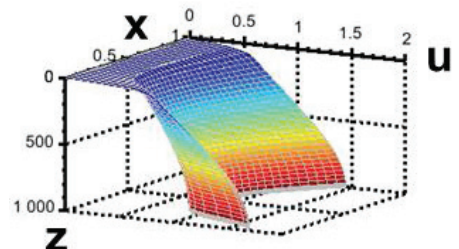

$\mathbf{Z}$

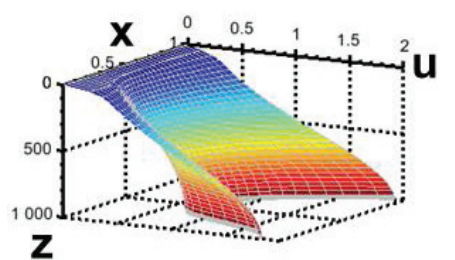

$(\mathrm{C}-2)$
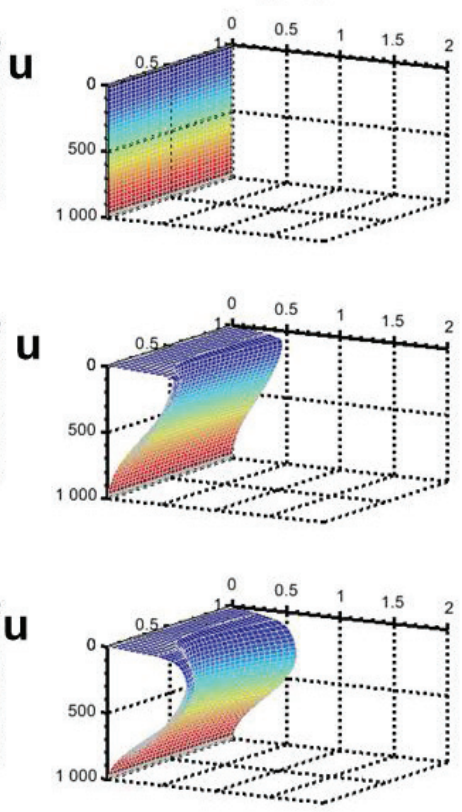

(a)

(C-3)

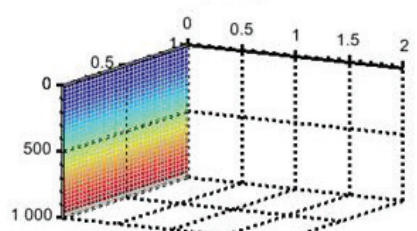

(b)

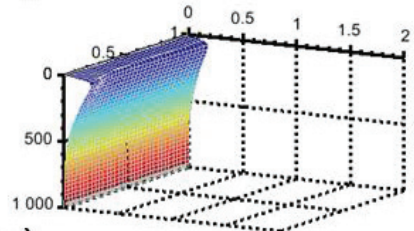

(c)

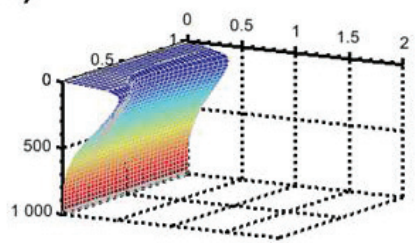

(C-4)
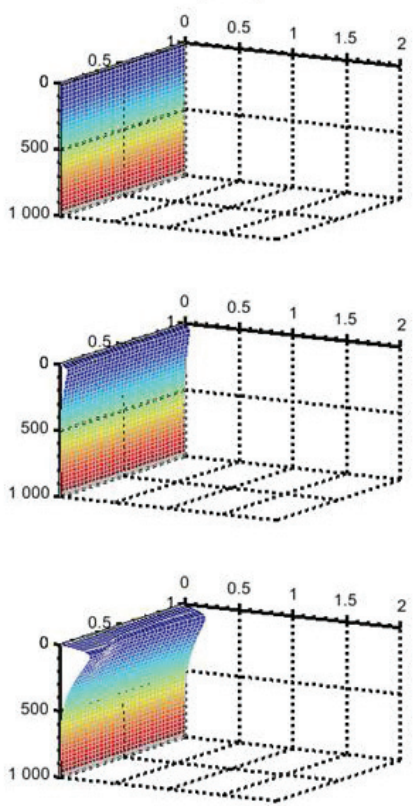

Figure 1. Plots of the population density in each compartment 1 through 4 ([C-1] through [C-4]) at (a) the start $t=0.0$, (b) middle $t=0.5$, and (c) end $t=1.0$ for $p_{0}=2.0$. In this case $N_{1}=N_{2}=L=32$. Note also that $u_{1}^{0}(x, z)=1$, and $u_{p}^{0}(x, z)=0$ for $p=2,3,4$ in (a).
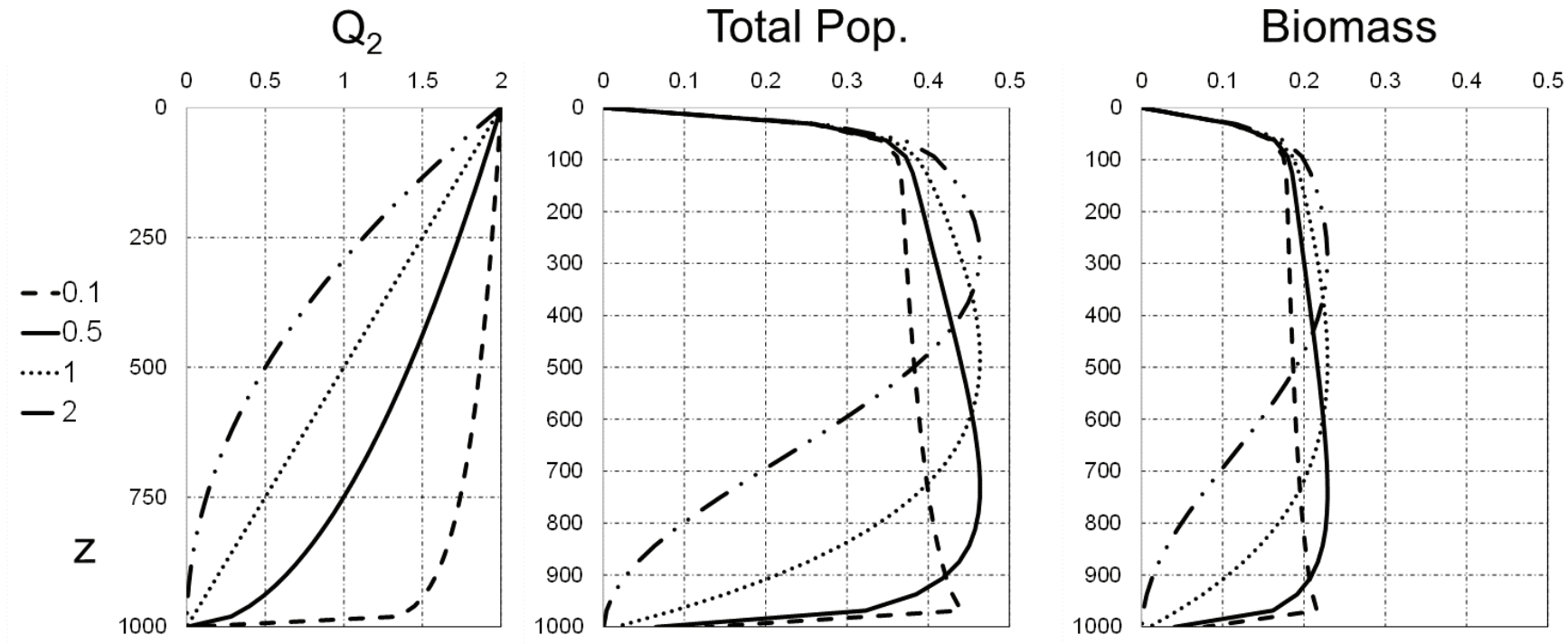

Figure 2. Vertical profiles of the transfer rates $Q_{2}(z)$, total population, and biomass for various values of the transfer exponent $p_{0}=0.1,0.5,1.0,2.0$ in $(\mathrm{C}-2)$ at time $t=1.0$.

For the exercise we consider a single phytoplankton species for the sake of simplicity but the analysis can be naturally extended to the multi-species case. The flow rate $Q_{p}$ between compartments decreases with depth and is constant in time given by

$$
Q_{p}=2.0\left(\frac{z_{\max }-z}{z_{\max }}\right)^{p_{0}}
$$

where we note that particles are allowed to freely exit the fourth compartment. The exponent $p_{0}$ plays the role of determining the rate of decay of the vertical flow profile with larger values of $p_{0}$ leading to higher 
advection rates (e.g., velocities) in the upper water layers. This may happen e.g., as a result of wind forcing on the ocean's surface or river currents encountering frictional resistance at the river bottom. Values of $p_{0}>0$ correspond with more rapid decay of the velocity current while values of $p_{0}<0$ lead to a more uniform velocity distribution with a sharp decline near the bottom layer. The individual growth rate follows a von-Bertalanffy type model and is given by

$$
g_{p}(x)=g_{\max }\left(x_{\max }-x\right),
$$

where $g_{\max }=1$ for each region. The sinking $w_{p}$ and reproduction rate $\beta_{p}$ of individuals is assumed to increase linearly with body size:

$$
w_{p}(x)=w_{\max } x, \quad \beta_{p}=\beta_{\max } x,
$$

where $w_{\max }=25.0, \beta_{\max }=0.4, F_{p}=0$, and assume that the effects of the environment $\vec{Y}$ are negligible. The mortality rate is taken as a constant $\mu_{p}=0.1$. The coagulation rate is assumed to be the same across each compartment and is taken to be proportional to the size of the colliding particles:

$$
\eta_{p}(x, s)=\eta_{\max }(p) s x
$$

for $x+s<x_{\max }$ and $\eta_{p}(x, s)=0$ for $x+s \geq x_{\max }$. The source term $S_{p}^{B}=0$ for each of the trials.

The total population sampled at depth $z$ is taken as $P_{p}(z)=\int_{0}^{1} u_{p} d x$ and the sampled biomass at depth $z$ is given by $B_{p}(z)=\int_{0}^{1} x u_{p} d x$ for regions $p=1, \ldots, 4$. In phytoplankton studies these population metrics are often directly correlated with important field measurements such as chlorophyll-a.

For the trials we varied the exponent for $p_{0}=0.1,0.5,1.0,2.0$ while holding all other parameters the same for each trial. We also kept a constant mesh ratio of $\Delta x=\Delta z=\Delta t$. The results indicate that flow concentration in the uppermost layers $p_{0}<1$ combined with particle settling has the effect of concentrating mass well below the water surface (Figures 1 and 2). As pointed out in [33] , it is well known that local maxima on the total population or biomass profiles are correlated with light and nutrient availability. Since these factors were not taken into account in the trials the results suggest that purely physical factors such as settling of particles combined with non-uniform surface currents could also play a meaningful role in the formation of DCM's. The interaction of various effects in fully-resolved phytoplankton models gives rise to interesting phenomena both from purely biological and mathematical standpoints [24]. Our trials suggest that the model is capable of replicating natural patterns and provides a reasonable tool for future applications.

\section{Conclusions}

We have established the well-posedness of a general model featuring coagulation and vertical effects in a multi-region setting. The model considered here is mathematically general and can be adapted to investigate particle coagulation problems in a wide variety of biological and engineering contexts (e.g., cloud physics [38]). Using Taylor approximations one can also show that the transfer terms in (2.1) approximate an advection-diffusion process as the regions become arbitrarily small. We also point out here that the nonlinear model does not include fragmentation effects such as those extensively analyzed by Banasiak and Lamb in microparticle contexts [1, 12, 13, 14]. Additionally, fragmentation/coagulation 
models have also been applied to study schooling behavior of fish (e.g., [34]), and the multi-region context is a natural setting to study these type of problems. Considering the preceding examples, the incorporation of explicit advection and diffusion and the treatment of fragmentation processes would make the model more realistic from a physical standpoint. A first-order scheme was applied in this paper so another notable enhancement would be the development of high-resolution numerical schemes for (2.1) which would also make the model more suitable for applications (e.g., [29]). Future efforts should also focus on size-specific field data collection and assimilation which are absolutely critical in the development of realistic sub-models of the various microbiological processes determining the fate of individuals.

\section{Acknowledgments}

The authors would like to thank two anonymous referees for suggestions that improved the manuscript. The research of A.S. Ackleh was supported in part by funds from the R.P. Authement Eminent Scholar and Endowed Chair in Computational Mathematics. The research of R. Miller was supported in part by the Louisiana Board of Regents Support Fund Grant LEQSF (2020-23)-RD-A-25.

\section{References}

[1] A.S. Ackleh, Estimation of parameters in a structured algal coagulation-fragmentation model, Nonlinear Analysis, Theory Methods and Applications, 28 (1997), 837-854.

[2] A.S. Ackleh, R. Ferdinand, A nonlinear phytoplankton aggregation model with light shading, SIAM Journal of Applied Mathematics, 60 (1999), 316-336.

[3] A.S. Ackleh, H.T. Banks, K. Deng, A finite difference approximation for a coupled system of nonlinear size-structured populations, Nonlinear Analysis, 50(6) (2002), 727-748.

[4] A.S. Ackleh, K. Deng, S. Hu, On a nonlinear size-structured phytoplankton-zoopankton aggregation model, Dynamics of Continuous, Discrete and Impulsive Systems Series A: Mathematical Analysis, 14(2) (2007), 265-285.

[5] A.S. Ackleh, K. Deng and X. Wang, Existence-uniqueness and monotone approximation for a phytoplanktonzooplankton aggregation model, Zeitschrift Angewandte Mathematik und Physik (ZAMP), 57 (2006), 733-749.

[6] A.S. Ackleh and B.G. Fitzpatrick, Modeling aggregation and growth processes in an algal population model: analysis and computation, Journal of Mathematical Biology, 35 (1997), 480-502.

[7] A.S. Ackleh, R. Miller, A model for the interaction of phytoplankton aggregates and the environment: approximation and parameter estimation, Inverse Problems in Science and Engineering, 26(2) (2017), 152-182.

[8] A.S. Ackleh, T.G. Hallam, W.O. Smith Jr., Influences of aggregation and grazing on phytoplankton dynamics and fluxes: an individual-based modeling approach, Nonlinear World 1 (1994), 473-492.

[9] A.S. Ackleh, K. Ito, An implicit finite difference scheme for the nonlinear size-structured population model, Numerical Functional Analysis and Optimization, 18 (1997), 865-884.

[10] A.S. Ackleh, B. Ma, R. Miller, A general nonlinear model for the interaction of a size-structured population and its environment: well-posedness and approximation, Quarterly of Applied Mathematics, 74 (2016), 671-704.

[11] M.E. Baird, P.R. Oke, I.M. Suthers, J.H. Middleton, A plankton population model with biomechanical descriptions of biological processes in an idealised 2D ocean basin, Journal of Marine Systems, 50 (2004), 199-222.

[12] J. Banasiak, Shattering and non-uniqueness in fragmentation models - an analytic approach, Physica D, 222 (2006), 63-72. 
[13] J. Banasiak, Transport processes with coagulation and strong fragmentation, Discrete and Continuous Dynamical Systems - Series B, 17(2) (2012), 445-472.

[14] J. Banasiak, W. Lamb, Coagulation, fragmentation, and growth processes in a size structured population, Discrete and Continuous Dynamical Systems - Series B, 11(3) (2009), 563-585.

[15] J. Droniou and R. Eymard, Uniform-in-time convergence of numerical methods for non-linear degenerate parabolic equations, Numerische Mathematik, 132 (4) (2016), 721-766.

[16] M. Estrada, C. Marrase, M. Latasa, E. Berdalet, M. Delgado, T. Riera, Variability of deep chlorophyll maximum characteristics in the Northwestern Mediterranean, Marine Ecology Progress Series, 92 (1993), 289-300.

[17] B. Faugeras, O. Maury, An advection-diffusion-reaction size-structured fish population dynamics model combined with a statistical parameter estimation procedure: application to the indian ocean skipjack tuna fishery, Mathematical Biosciences and Engineering, 2:4 (2005), 1-23.

[18] B. Faugeras, O. Maury, A multi-region nonlinear age-size structured fish population model, Nonlinear Analysis: Real World Applications, 6(3) (2005), 447-460.

[19] K. Fennel, R. Hetland, Y. Feng, S. DiMarco, A coupled physical-biological model of the Northern Gulf of Mexico shelf: model description, validation and analysis of phytoplankton variability, Biogeosciences, 8 (2011), 1881-1899.

[20] R.R. Ferdinand, A nonlinear coupled phytoplankton dynamics model, Numerical Functional Analysis and Optimization, 23:5-6 (2002), 515-528.

[21] W.W. Gregg, P. Ginoux, P.S. Schopf, N.W. Casey, Phytoplankton and iron: validation of a global three-dimensional ocean biogeochemical model, Deep Sea Research II, 50 (2003), 3143-3169.

[22] J.M. Hamrick, T.S. Wu, Computational design and optimization of the EFDC/HEM3D surface water hydrodynamic and eutrophication models, Proceedings of the NGEMCOM workshop at the National Environmental Supercomputing Center, Bay City, MI, (August 7-9, 1995), 143-156.

[23] S. Hinckley, K.O. Coyle, G. Gibson, A.J. Hermann, E.L. Dobbins, A biophysical NPZ model with iron for the Gulf of Alaska: reproducing the differences between an oceanic HNLC ecosystem and a classical northern temperate shelf ecosystem, Deep Sea Research II, 56 (2009), 2520-2536.

[24] J. Huisman, N.N. Pham Thi, D.M. Karl, B. Sommeijer, Reduced mixing generates oscillations and chaos in the oceanic deep chlorophyll maximum, Nature Letters, 439 (2006), doi:10.1038/nature04242.

[25] G.A. Jackson, S.E. Lochmann, Effect of coagulation on nutrient and light limitation of an algal bloom, Limnology and Oceanography, 37(1) (1992), 77-89.

[26] G.A. Jackson, A.M. Waite, P.W. Boyd, Role of algal aggregation in vertical carbon export during SOIREE and in other low biomass environments, Geophysical Research Letters, 32 (2005), 1-4.

[27] T. Kiørboe, Small-scale turbulence, marine snow formation, and planktivorous feeding, Scientia Marina, 61 (Supl.1) (1997), 141-158.

[28] M. Latasa, A.M. Cabello, X. Moran, R. Massana, R. Scharek, Distribution of phytoplankton groups within the deep chlorophyll maximum, Limnology and Oceanography, 62 (2017), 665-685.

[29] E.C. Laurenceau-Cornec, T.W. Trull, D.M. Davies, S.G. Bray, J.Doran, F. Planchon, F. Carlotti, M.-P. Jouandet, A.-J. Cavagna, A.M. Waite, S. Blain, The relative importance of phytoplankton aggregates and zooplankton fecal pellets to carbon export: insights from free-drifting sediment trap deployments in naturally iron-fertilised waters near the Kergeulen Plateau, Biogeosciences, 12 (2015), 1007-1027.

[30] J.H. Martin, R.M. Gordon, S.E. Fitzwater, The case for iron, Limnology and Oceanography, 36(8) (1991), 1793-1802.

[31] C.B. Miller, B.W. Frost, B.B. Booth, P.A. Wheeler, M.R. Landry, N. Welschmeyer, Ecological processes in the subarctic Pacific: iron limitation cannot be the whole story, Oceanography, 4(2) (1991), 71-78.

[32] M.M. Mills, C. Ridame, M. Davey, J. La Roche, R.J. Geider, Iron and phosphorous co-limit nitrogen fixation in the eastern tropical North Atlantic, Nature, 429 (2004), 292-294. 
[33] H.V. Moeller, C. Laufkotter, E.M. Sweeney, M.D. Johnson, Light-dependent grazing can drive formation and deepening of deep chlorophyll maxima, Nature Communications, 10, 1978 (2019). https://doi.org/10.1038/s41467-01909591-2.

[34] H-S Niwa, School Size Statistics of Fish, Journal of Theoretical Biology, 195 (1998), 351-361.

[35] S.C. Noutchie, Analysis of the effects of fragmentation-coagulation in planktology, Comptes Rendus Biologies, 333 (2010), 789-792.

[36] B. Penta, D. Ko, R. Gould, R. Arnone, R. Greene, J. Lehrter, J. Hagy, B.Scheffer, M. Murrell, J. Kurtz, B. Herchenroder, R. Green, P. Eldridge, Using coupled models to study the effects of river discharge on biogeochemical cycling and hypoxia in the northern Gulf of Mexico, Oceans '09 MTS/IEEE Conference, (2009).

[37] U. Riebesell, Particle aggregation during a diatom bloom, Marine Ecology Progress Series, 69 (1991), 281-291.

[38] Y. Sato, T. Nakajima, K. Suzuki, T. Iguchi, Application of a Monte Carlo integration method to collision and coagulation growth processes of hydrometeors in a bin-type model, Journal of Geophysical Research, 114(D9) (2009), $1-19$

[39] J. Smoller, Shock waves and reaction-diffusion equations, Second Ed., Springer-Verlag (1994).

[40] W. Sunda, S.A. Huntsman, Interrelated influence of iron, light and cell size on marine phytoplankton growth, Nature, 390 (1997), 389-392.

[41] K.R. Timmermans, L.J.A. Gerringa, H.J.W. de Baar, B. van der Wagt, M.J.W. Veldhuis, J.T.M. de Jong, P.L. Croot, Growth rates of large and small Southern Ocean diatoms in relation to availability of iron in natural seawater, Limnology and Oceanography, 46(2) (2001), 260-266.

[42] Z. Xue, R. He, K. Fennel, W.-J. Cai, S. Lohrenz, C. Hopkinson, Modeling ocean circulation and biogeochemical variability in the Gulf of Mexico, Biogeosciences, 10 (2013), 7219-7234.

\section{Appendix: Proof of lemmas}

Proof of Lemma 5.2. Applying Lemma 5.1 and an approach similar to that one taken in the $\ell_{1}$ proof in [7] we obtain,

$$
\begin{aligned}
\left\|u_{p}^{k+1}\right\|_{1} \leq & \left\|u_{p}^{k}\right\|_{1}+\left(c_{1} x_{\max } z_{\max }+c_{1}\|\theta\|_{\infty}\left\|\vec{u}^{k}\right\|_{1}+c_{1} x_{\max } z_{\max }\left\|\vec{Y}^{k}\right\|_{\infty}\right) \Delta t \\
& +\|Q\|_{\infty}\left\|\vec{u}^{k}\right\|_{1} \Delta t+\left(c_{1} z_{\max }+c_{1} z_{\max }\left\|\vec{Y}^{k}\right\|_{\infty}+\|\beta\|_{\infty}\left\|\vec{u}^{k}\right\|_{1}\right) \Delta t+c_{0} x_{\max } \Delta t .
\end{aligned}
$$

Next by (4.3), assumption (B3) and non-negativity of the numerical scheme we have

$$
\begin{aligned}
\left|Y_{p, \ell, l}^{k+1}\right|=Y_{p, \ell, l}^{k+1} & \leq Y_{p, \ell, l}^{k}+f_{p, \ell, l}^{k} \Delta t+\sum_{i=1}^{n} \mathcal{Q}_{p, i, \ell, l}^{k} Y_{i, \ell, l}^{k} \Delta t \\
& \leq Y_{p, \ell, l}^{k}+\left(c_{1}+c_{1} c_{0}\left\|\vec{u}^{k}\right\|_{1}+c_{1}\left\|\vec{Y}^{k}\right\|_{\infty}\right) \Delta t+\|\mathcal{Q}\|_{\infty}\left\|\vec{Y}^{k}\right\|_{\infty} \Delta t \\
& \leq\left\|\vec{Y}^{k}\right\|_{\infty}+\left(c_{1}+c_{1} c_{0}\left\|\vec{u}^{k}\right\|_{1}+c_{1}\left\|\vec{Y}^{k}\right\|_{\infty}\right) \Delta t+\|\mathcal{Q}\|_{\infty}\left\|\vec{Y}^{k}\right\|_{\infty} \Delta t \\
& \leq\left(1+\left(c_{1}+\|\mathcal{Q}\|_{\infty}\right) \Delta t\right)\left\|\vec{Y}^{k}\right\|_{\infty}+c_{1} c_{0}\left\|\vec{u}^{k}\right\|_{1} \Delta t
\end{aligned}
$$

and from the preceding inequality and assumption (B3) we get

$$
\left\|\vec{Y}^{k+1}\right\|_{\infty} \leq\left(1+\left(c_{1}+c_{0}\right) m n \Delta t\right)\left\|\vec{Y}^{k}\right\|_{\infty}+c_{1} c_{0} m n\left\|\vec{u}^{k}\right\|_{1} \Delta t .
$$

Summing (9.1) over $p=1, \ldots, n$ and adding this result to (9.2) we arrive at

$$
\left\|\vec{u}^{k+1}\right\|_{1}+\left\|\vec{Y}^{k+1}\right\|_{\infty} \leq\left(1+C_{1,1} \Delta t\right)\left\|\vec{u}^{k}\right\|_{1}+\left(1+C_{1,2} \Delta t\right)\left\|\vec{Y}^{k}\right\|_{\infty}+C_{1,3} \Delta t,
$$

for suitably large positive constants $C_{1,1}, C_{1,2}$ and $C_{1,3}$. The final result can be established from (9.3) by induction and elementary calculations. 
Proof of Lemma 5.3. Define

$$
u_{p, j_{0}, l_{0}}^{k+1}=\max _{j=0, \ldots, N ; l=0, \ldots, R}\left(u_{p, j, l}^{k+1}\right)=\left\|u_{p}^{k+1}\right\|_{\infty} .
$$

If $j_{0}=0$ or $l_{0}=0$ substituting the boundary conditions at the corner and the free surface respectively, and appealing to assumptions (B1), (B4), (B5), and Lemma 5.2,

$$
\begin{aligned}
\sum_{p=1}^{n}\left\|u_{p}^{k+1}\right\|_{\infty} & \leq n \max \left\{\frac{\left(1+B_{1}\right) \max \left\{c_{1},\|\beta\|_{\infty}\right\}}{\zeta_{1}}, \frac{\|\alpha\|_{\infty}}{\zeta_{2}}\right\} \\
& \leq n \max \left\{\frac{\left(1+B_{1}\right) \max \left\{c_{1}, c_{0}\right\}}{\zeta_{1}}, \frac{c_{0}}{\zeta_{2}}\right\}=B_{2,1}
\end{aligned}
$$

Otherwise, fix $j_{0} \geq 1$ and $l_{0} \geq 1$. Using the fact $u_{p, j_{0}, l_{0}}^{k+1}-u_{p, j_{0}-1, l_{0}}^{k+1} \geq 0$, and $u_{p, j_{0}, l_{0}}^{k+1}-u_{p, j_{0}, l_{0}-1}^{k+1} \geq 0$ and invoking nonnegativity of the scheme and vital rate parameters we have

$$
\begin{aligned}
& \left(1+\Delta t\left(\mu_{p, j_{0}, l_{0}}^{k}+\frac{g_{p, j_{0}, l_{0}}^{k}-g_{p, j_{0}-1, l_{0}}^{k}}{\Delta x}+\frac{w_{p, j_{0}, l_{0}}^{k}-w_{p, j_{0}, l_{0}-1}^{k}}{\Delta z}\right)\right) u_{p, j_{0}, l_{0}}^{k+1} \\
\leq & u_{p, j_{0}, l_{0}}^{k}+\frac{\Delta t}{2} \sum_{s=1}^{j_{0}-1} \eta_{p, j_{0}-s, s, l_{0}}^{k} u_{p, j_{0}-s, l_{0}}^{k} u_{p, s, l_{0}}^{k+1} \Delta x+\Delta t S_{p, j_{0}, l_{0}}^{B, k}+\Delta t \sum_{i=1}^{n} Q_{p, i, j_{0}, l_{0}}^{k} u_{i, j_{0}, l_{0}}^{k} .
\end{aligned}
$$

Applying (5.1) we obtain

$$
(1-\lambda \Delta t)\left\|u_{p}^{k+1}\right\|_{\infty} \leq\left\|u_{p}^{k}\right\|_{\infty}+\frac{\Delta t}{2}\|\eta\|_{\infty}\left\|u_{p}^{k}\right\|_{1}\left\|u_{p}^{k+1}\right\|_{\infty}+\Delta t\left\|S_{p}^{B, k}\right\|_{\infty}+\Delta t\|Q\|_{\infty} \sum_{p=1}^{n}\left\|u_{p}^{k}\right\|_{\infty} .
$$

Setting $C_{\infty}=\max \left\{0.5 c_{0} B_{1}, \lambda\right\}$, noting that $1-C_{\infty} \Delta t>0$, and summing from $p=1, \ldots, n$ we arrive at

$$
\sum_{p=1}^{n}\left\|u_{p}^{k+1}\right\|_{\infty} \leq\left(\frac{1+c_{0} \Delta t}{1-C_{\infty} \Delta t}\right) \sum_{p=1}^{n}\left\|u_{p}^{k}\right\|_{\infty}+\frac{n B_{1,1} \Delta t}{1-C_{\infty} \Delta t}
$$

The final result follows from induction and assumption (B7).

Proof of Lemma 5.4. The first two estimates in (5.4.1) follow directly from the methods used in [7] so we omit the details. Towards proving the third estimate in (5.4.1) from the scheme, and assumption (B3) we obtain

$$
\begin{aligned}
\left|Y_{p, \ell, l}^{k+1}-Y_{p, \ell, l-1}^{k+1}\right| \leq & \left(1+\Delta t\left(\sum_{i=1}^{n} \mathcal{Q}_{i, p, \ell, l-1}^{k}+h_{p, \ell, l-1}^{k}\right)\right)\left|Y_{p, \ell, l}^{k+1}-Y_{p, \ell, l-1}^{k+1}\right| \\
\leq & \left|Y_{p, \ell, l}^{k}-Y_{p, \ell, l-1}^{k}\right|+\Delta t c_{0} \sum_{i=1}^{n}\left|Y_{i, \ell, l}^{k}-Y_{i, \ell, l-1}^{k}\right| \\
& +2 B_{2} L_{\mathcal{Q}} \sum_{i=1}^{n}\left(\Delta z+\sum_{p=1}^{n} \sum_{\ell=1}^{m}\left|\sigma_{p, \ell, l}^{k}-\sigma_{p, \ell, l-1}^{k}\right|+\sum_{p=1}^{n}\left|Y_{p, \ell, l}^{k}-Y_{p, \ell, l-1}^{k}\right|\right) \Delta t \\
& +\Delta t L_{f}\left(\Delta z+\sum_{p=1}^{n} \sum_{\ell=1}^{m}\left|\sigma_{p, \ell, l}^{k}-\sigma_{p, \ell, l-1}^{k}\right|+\sum_{p=1}^{n}\left|Y_{p, \ell, l}^{k}-Y_{p, \ell, l-1}^{k}\right|\right) \Delta t \\
& +\Delta t B_{2} L_{h}\left(\Delta z+\sum_{p=1}^{n} \sum_{\ell=1}^{m}\left|\sigma_{p, \ell, l}^{k}-\sigma_{p, \ell, l-1}^{k}\right|+\sum_{p=1}^{n}\left|Y_{p, \ell, l}^{k}-Y_{p, \ell, l-1}^{k}\right|\right) \Delta t .
\end{aligned}
$$

Let $B_{3,1}=n m\left(2 n B_{2} L_{\mathcal{Q}}+L_{f}+B_{2} L_{h}+c_{0}\right)\left(1+B_{3}\right)$ and applying the second estimate in (5.4.1) and summing over $p=1, \ldots, n$ we obtain the following recurrence relationship:

$$
\sum_{p=1}^{n} \sum_{\ell=1}^{m}\left|Y_{p, \ell, l}^{k+1}-Y_{p, \ell, l-1}^{k+1}\right| \leq\left(1+B_{3,1} \Delta t\right) \sum_{p=1}^{n} \sum_{\ell=1}^{m}\left|Y_{p, \ell, l}^{k}-Y_{p, \ell, l-1}^{k}\right|+B_{3,1} \Delta z \Delta t .
$$

Applying elementary calculations and induction on $k$ we have the following

$$
\sum_{p=1}^{n} \sum_{\ell=1}^{m}\left|Y_{p, \ell, l}^{k+1}-Y_{p, \ell, l-1}^{k+1}\right| \leq e^{B_{3,1} T}\left(\sum_{p=1}^{n} \sum_{\ell=1}^{m}\left|Y_{p, \ell, l}^{0}-Y_{p, \ell, l-1}^{0}\right|+B_{3,1} T \Delta z\right) \leq B_{3,1,1} \Delta z .
$$

where the last step follows from the Lipschitz condition on the initial condition (B7) with $B_{3,1,1}=$ $\exp \left(B_{3,1} T\right)\left(n m L_{Y^{0}}+B_{3,1} T\right)$. This proves estimate (5.4.1).

We omit the details for the constants $B_{3,2}, \ldots, B_{3,7}$, since the results follow directly from the Lipschitz assumptions on the model parameters, estimate (5.4.1), Lemma 5.2, and the arguments presented in 
Lemma 5.3 in [10]. Towards proving the estimates in (5.4.8) first observe that by 4.1, for any $j=$ $1, \ldots, N$ and $l=1, \ldots, R$

$$
\begin{aligned}
\left(u_{p, j, l}^{k+1}-u_{p, j, l}^{k}\right)= & \frac{\Delta t}{2} \sum_{s=1}^{j-1} \eta_{p, j-s, s, l}^{k} u_{p, j-s, l}^{k} u_{p, s, l}^{k+1} \Delta x+\Delta t S_{p, j, l}^{B, k}+\Delta t \sum_{i=1}^{n} Q_{p, i, j, l}^{k} u_{i, j, l}^{k} \\
& -\frac{\Delta t}{\Delta x}\left(\left(g_{p, j, l}^{k}-g_{p, j-1, l}^{k}\right) u_{p, j, l}^{k+1}+\left(u_{p, j, l}^{k+1}-u_{p, j-1, l}^{k+1}\right) g_{p, j-1, l}^{k}\right) \\
& -\frac{\Delta t}{\Delta z}\left(\left(w_{p, j, l}^{k}-w_{p, j, l-1}^{k}\right) u_{p, j, l}^{k+1}+\left(u_{p, j, l}^{k+1}-u_{p, j, l-1}^{k+1}\right) w_{p, j, l-1}^{k}\right) \\
& -\Delta t \mu_{p, j, l}^{k} u_{p, j, l}^{k+1}-\Delta t \sum_{i=1}^{n} Q_{i, p, j, l}^{k} u_{p, j, l}^{k+1}-\Delta t u_{p, j, l}^{k+1} \sum_{q=1}^{N} \eta_{p, q, j, l}^{k} u_{p, q, l}^{k} \Delta x .
\end{aligned}
$$

For the first estimate in (5.4.8) applying the boundary condition, the triangle inequality and preceding estimates we have

$$
\begin{aligned}
g_{p, 0, l}^{k}\left|u_{p, 0, l+1}^{k+1}-u_{p, 0, l}^{k+1}\right| \leq & B_{3,2} \Delta z+B_{2} B_{3,2} \Delta z+B_{3,2} \sum_{i=1}^{n} \sum_{j=1}^{N} u_{i, j, l+1}^{k} \Delta x \Delta z \\
& +c_{0} \sum_{i=1}^{n} \sum_{j=1}^{N}\left|u_{i, j, l+1}^{k}-u_{i, j, l}^{k}\right| \Delta x
\end{aligned}
$$

and thus

$$
\sum_{l=1}^{R-1} \frac{\Delta t}{\Delta x}\left|u_{p, 0, l+1}^{k+1}-u_{p, 0, l}^{k+1}\right| g_{p, 0, l}^{k} \Delta x \leq B_{3,8} \Delta t\left(1+T V_{z}\left(\vec{u}^{k}\right)\right) .
$$

where $B_{3,8} \geq \max \left\{c_{0}, B_{3,2} z_{\max }+B_{2} B_{3,2} z_{\max }+B_{3,2} B_{1}\right\}$. This establishes the first estimate in (5.4.8). For the second estimate note that for all $j=0, \ldots, N-1$ we have

$$
\left(u_{p, j+1,0}^{k+1}-u_{p, j, 0}^{k+1}\right) w_{p, j, 0}^{k}=\left(\alpha_{p, j+1}^{k}-\alpha_{p, j}^{k}\right)-\left(w_{p, j+1,0}^{k}-w_{p, j, 0}^{k}\right) u_{p, j+1,0}^{k+1} .
$$

and by estimate (5.4.3) we have

$$
\begin{aligned}
\frac{\Delta t}{\Delta z}\left|u_{p, j+1,0}^{k+1}-u_{p, j, 0}^{k+1}\right| w_{p, j, 0}^{k} \Delta z & \leq\left|\alpha_{p, j+1}^{k}-\alpha_{p, j}^{k}\right| \Delta t+\left|w_{p, j+1,0}^{k}-w_{p, j, 0}^{k}\right| u_{p, j+1,0}^{k+1} \Delta t \\
& \leq B_{3,3}\left(1+B_{2}\right) \Delta x \Delta t .
\end{aligned}
$$

Summing over $j=1, \ldots, N$ and enlarging $B_{3,8}$ as necessary we obtain the second estimate:

$$
\sum_{j=1}^{N} \frac{\Delta t}{\Delta z}\left|u_{p, j+1,0}^{k+1}-u_{p, j, 0}^{k+1}\right| w_{p, j, 0}^{k} \Delta z \leq B_{3,3}\left(1+B_{2}\right) x_{\max } \Delta t \leq B_{3,8} \Delta t .
$$

Towards proving the third estimate in (5.4.8) we have

$$
\begin{aligned}
\left(u_{p, 1, l}^{k+1}-u_{p, 0, l}^{k+1}\right) & =\left(u_{p, 1, l}^{k+1}-u_{p, 1, l}^{k}\right)+\left(u_{p, 1, l}^{k}-u_{p, 0, l}^{k}\right)+\left(u_{p, 0, l}^{k}-u_{p, 0, l}^{k+1}\right) \\
& =\left(A_{1}\right)+\left(A_{2}\right)+\left(A_{3}\right) .
\end{aligned}
$$

Applying (9.5) to expand $\left(A_{1}\right)$ in 9.7, applying the triangle inequality, and (9.6), multiplying by $\Delta z$ and summing over $l=1, \ldots, R$ we get

$$
\begin{aligned}
& \sum_{l=1}^{R}\left(1+\frac{\Delta t}{\Delta x} g_{p, 0, l}^{k}\right)\left|u_{p, 1, l}^{k+1}-u_{p, 0, l}^{k+1}\right| \Delta z+\Delta t w_{p, 1, R}^{k}\left|u_{p, 1, R}^{k+1}-u_{p, 0, R}^{k+1}\right|-\Delta t\left|u_{p, 0,0}^{k+1}-u_{p, 1,0}^{k+1}\right| w_{p, 1,0}^{k} \\
& \leq \frac{\Delta t}{2} c_{0} B_{2} B_{1}+\Delta t\left(B_{1,1}+n c_{0} B_{2}+B_{3,3} B_{2}+B_{3,2} B_{2}\right) z_{\max } \\
& +\Delta t \frac{B_{1,1}}{\zeta_{1}}\left(B_{3,2} z_{\max }+B_{2} B_{3,2} z_{\max }+B_{3,2} B_{1} z_{\max }+c_{0} T V_{x}\left(\vec{u}^{k}\right)\right) \\
& +\Delta t B_{3,2} \sum_{l=1}^{R}\left|u_{p, 0, l-1}^{k+1}-u_{p, 1, l-1}^{k+1}\right| \Delta z+\Delta t B_{1,1} B_{2} z_{\max }+\Delta t n c_{0} B_{2} z_{\max } \\
& +\Delta \operatorname{tnc}_{0} B_{2} B_{1}+\sum_{l=1}^{R}\left|u_{p, 1, l}^{k}-u_{p, 0, l}^{k}\right| \Delta z+\sum_{l=1}^{R}\left|u_{p, 0, l}^{k}-u_{p, 0, l}^{k+1}\right| \Delta z
\end{aligned}
$$

thereby establishing existence of a suitably large constant $B_{3,8,2}$ such that

$$
\begin{aligned}
& \sum_{l=1}^{R}\left(1+\frac{\Delta t}{\Delta x} g_{p, 0, l}^{k}\right)\left|u_{p, 1, l}^{k+1}-u_{p, 0, l}^{k+1}\right| \Delta z \leq B_{3,8,2}\left(1+x_{\max }+T V_{x}\left(\vec{u}^{k}\right)\right) \Delta t \\
& \quad+\Delta t B_{3,2} \sum_{l=1}^{R}\left|u_{p, 0, l}^{k+1}-u_{p, 1, l}^{k+1}\right| \Delta z+\sum_{l=1}^{R}\left|u_{p, 1, l}^{k}-u_{p, 0, l}^{k}\right| \Delta z+\sum_{l=1}^{R}\left|u_{p, 0, l}^{k}-u_{p, 0, l}^{k+1}\right| \Delta z
\end{aligned}
$$


where we used the estimate $\left|u_{p, 1,0}^{k+1}-u_{p, 0,0}^{k+1}\right| w_{p, 1,0}^{k} \leq\left(L_{\alpha}+L_{w} B_{2}\right) \Delta x$. Expanding $\left(A_{3}\right)$, applying the scheme (4.1), the triangle inequality, and Lemma 5.3, estimates (5.4.6) and (5.4.7) we have

$$
\begin{aligned}
& \left|u_{p, 0, l}^{k+1}-u_{p, 0, l}^{k}\right|=\mid \frac{1}{g_{p, 0, l}^{k}}\left(F_{p, l}^{k}-F_{p, l}^{k-1}\right)-\frac{1}{g_{p, 0, l}^{k}}\left(g_{p, 0, l}^{k}-g_{p, 0, l}^{k-1}\right) u_{p, 0, l}^{k} \\
& +\frac{1}{g_{p, 0, l}^{k}} \sum_{i=1}^{n} \sum_{j=1}^{N}\left(\beta_{p, i, j, l}^{k}-\beta_{p, i, j, l}^{k-1}\right) u_{i, j, l}^{k} \Delta x+\frac{1}{g_{p, 0, l}^{k}} \sum_{i=1}^{n} \sum_{j=1}^{N}\left(u_{i, j, l}^{k}-u_{i, j, l}^{k-1}\right) \beta_{p, i, j, l}^{k-1} \Delta x \mid \\
& \leq \frac{1}{\zeta_{1}} B_{3,7} \Delta t+\frac{1}{\zeta_{1}} B_{3,6} B_{2} \Delta t+\frac{B_{3,7} B_{2} \Delta t}{\zeta_{1}} n x_{\max } \\
& +\frac{c_{0}}{\zeta_{1}} n x_{\max }\left(1.5 c_{0} B_{2}^{2} x_{\max }+B_{1,1}+2 n c_{0} B_{2}+B_{3,3} B_{2}+B_{3,2} B_{2}+B_{1,1} B_{2}\right) \Delta t \\
& +\sum_{i=1}^{n} \sum_{j=1}^{N} \frac{\Delta t}{\Delta x}\left|u_{i, j, l}^{k}-u_{i, j-1, l}^{k}\right| B_{1,1} \Delta x+\sum_{i=1}^{n} \sum_{j=1}^{N} \frac{\Delta t}{\Delta z}\left|u_{i, j, l}^{k}-u_{i, j, l-1}^{k}\right| B_{1,1} \Delta x \\
& \leq B_{3,8,3}\left(\Delta t+\sum_{i=1}^{n} \sum_{j=1}^{N} \frac{\Delta t}{\Delta x}\left|u_{i, j, l}^{k}-u_{i, j-1, l}^{k}\right| \Delta x+\sum_{i=1}^{n} \sum_{j=1}^{N} \frac{\Delta t}{\Delta z}\left|u_{i, j, l}^{k}-u_{i, j, l-1}^{k}\right| \Delta x\right) .
\end{aligned}
$$

where $B_{3,8,3} \geq \max \left\{B_{3,7}+B_{3,6} B_{2}+B_{3,7} B_{2} n x_{\max }+c_{0} n x_{\max }\left(1.5 c_{0} B_{2}^{2} x_{\max }+B_{1,1}+2 n c_{0} B_{2}+B_{3,3} B_{2}+\right.\right.$ $\left.\left.B_{3,2} B_{2}+B_{1,1} B_{2}\right), B_{1,1}\right\} / \zeta_{1}$. Multiplying by $\Delta z$ and summing over $l=1, \ldots, R$ we have,

$$
\sum_{l=1}^{R}\left|u_{p, 0, l}^{k+1}-u_{p, 0, l}^{k}\right| \Delta z \leq B_{3,8,3}\left(z_{\max }+T V_{x}\left(\vec{u}^{k}\right)+T V_{z}\left(\vec{u}^{k}\right)\right) \Delta t .
$$

Combining the preceding estimate with (9.9) we get

$$
\begin{aligned}
& \quad \sum_{l=1}^{R}\left(1-B_{3,2} \Delta t+\frac{\Delta t}{\Delta x} g_{p, 0, l}^{k}\right)\left|u_{p, 1, l}^{k+1}-u_{p, 0, l}^{k+1}\right| \Delta z \leq B_{3,8,2}\left(1+x_{\max }+T V_{x}\left(\vec{u}^{k}\right)\right) \Delta t \\
& \quad+\sum_{l=1}^{R}\left|u_{p, 1, l}^{k}-u_{p, 0, l}^{k}\right| \Delta z+B_{3,8,3}\left(z_{\max }+T V_{x}\left(\vec{u}^{k}\right)+T V_{z}\left(\vec{u}^{k}\right)\right) \Delta t \\
& \leq B_{3,8}\left(1+T V_{x}\left(\vec{u}^{k}\right)+T V_{z}\left(\vec{u}^{k}\right)\right) \Delta t+\sum_{l=1}^{R}\left|u_{p, 1, l}^{k}-u_{p, 0, l}^{k}\right| \Delta z,
\end{aligned}
$$

where $B_{3,8}$ is enlarged as necessary so that $B_{3,8} \geq 2 \max \left\{B_{3,8,2}, B_{3,8,3}\right\}\left(x_{\max }+z_{\max }\right)$.

Towards proving the last estimate in (5.4.8) we have

$$
\left(u_{p, j, 1}^{k+1}-u_{p, j-1,1}^{k+1}\right)=\left(u_{p, j, 1}^{k+1}-u_{p, j, 0}^{k+1}\right)+\left(u_{p, j, 0}^{k+1}-u_{p, j-1,0}^{k+1}\right)+\left(u_{p, j-1,0}^{k+1}-u_{p, j-1,1}^{k+1}\right)
$$

and

$$
\left(u_{p, j, 1}^{k+1}-u_{p, j, 0}^{k+1}\right)=\left(u_{p, j, 1}^{k+1}-u_{p, j, 1}^{k}\right)+\left(u_{p, j, 1}^{k}-u_{p, j, 0}^{k}\right)+\left(u_{p, j, 0}^{k}-u_{p, j, 0}^{k+1}\right) .
$$

By (9.5) and (9.13), adding and subtracting terms we have

$$
\begin{aligned}
\left(u_{p, j, 1}^{k+1}-u_{p, j, 1}^{k}\right)= & \frac{\Delta t}{2} \sum_{s=1}^{j-1} \eta_{p, j-s, s, 1}^{k} u_{p, j-s, 1}^{k} u_{p, s, 1}^{k+1} \Delta x+\Delta t S_{p, j, 1}^{B, k}+\Delta t \sum_{i=1}^{n} Q_{p, i, j, 1}^{k} u_{i, j, 1}^{k} \\
& -\frac{\Delta t}{\Delta x}\left(\left(g_{p, j, 1}^{k}-g_{p, j-1,1}^{k}\right) u_{p, j-1,1}^{k+1}+\left(u_{p, j, 1}^{k+1}-u_{p, j, 0}^{k+1}\right) g_{p, j, 1}^{k}\right) \\
& -\frac{\Delta t}{\Delta x}\left(u_{p, j, 0}^{k+1}-u_{p, j-1,0}^{k+1}\right) g_{p, j, 1}^{k}-\frac{\Delta t}{\Delta x}\left(u_{p, j-1,0}^{k+1}-u_{p, j-1,1}^{k+1}\right)\left(g_{p, j, 1}^{k}-g_{p, j-1,1}^{k}\right) \\
& -\frac{\Delta t}{\Delta x}\left(u_{p, j-1,0}^{k+1}-u_{p, j-1,1}^{k+1}\right) g_{p, j-1,1}^{k}-\frac{\Delta t}{\Delta z}\left(\left(w_{p, j, 1}^{k}-w_{p, j, 0}^{k}\right) u_{p, j, 1}^{k+1}+\left(u_{p, j, 1}^{k+1}-u_{p, j, 0}^{k+1}\right) w_{p, j, 0}^{k}\right) \\
& -\Delta t \mu_{p, j, 1}^{k} u_{p, j, 1}^{k+1}-\Delta t \sum_{i=1}^{n} Q_{i, p, j, 1}^{k} u_{p, j, 1}^{k+1}-\Delta t u_{p, j, 1}^{k+1} \sum_{q=1}^{N} \eta_{p, q, j, 1}^{k} u_{p, q, 1}^{k} \Delta x
\end{aligned}
$$

Applying the preceding expansion and the triangle inequality to (9.14), then multiplying by $\Delta x$ and summing over $j=1, \ldots, N$ we obtain

$$
\begin{aligned}
& \sum_{j=1}^{N}\left(1+\frac{\Delta t}{\Delta z} w_{p, j, 0}^{k}\right)\left|u_{p, j, 1}^{k+1}-u_{p, j, 0}^{k+1}\right| \Delta x \leq 1.5 c_{0} B_{2}^{2} x_{\max }^{2} \Delta t \\
& \quad+\frac{B_{3,3}}{\zeta_{2}}\left(1+B_{2}\right) x_{\max } \Delta t+\Delta t B_{3,3} \sum_{j=1}^{N}\left|u_{p, j-1,0}^{k+1}-u_{p, j-1,1}^{k+1}\right| \Delta x \\
& \quad+\left(B_{1,1}+n c_{0} B_{2}+B_{3,3} B_{2}+B_{3,2} B_{2}+B_{1,1} B_{2}+n c_{0} B_{2}\right) x_{\max } \Delta t \\
& \quad+\frac{\Delta t}{\Delta x}\left|u_{p, 0,0}^{k+1}-u_{p, 0,1}^{k+1}\right| g_{p, 0,1}^{k} \Delta x+\sum_{j=1}^{N}\left|u_{p, j, 1}^{k}-u_{p, j, 0}^{k}\right| \Delta x+\sum_{j=1}^{N}\left|u_{p, j, 0}^{k}-u_{p, j, 0}^{k+1}\right| \Delta x .
\end{aligned}
$$

Note that $g_{p, 0,0}^{k} u_{p, 0,0}^{k+1}=g_{p, 0,0}^{k}\left(\alpha_{p}^{k} / w_{p, 0,0}^{k}\right)$ and applying the triangle inequality we obtain the following estimate

$$
\left|u_{p, 0,0}^{k+1}-u_{p, 0,1}^{k+1}\right| g_{p, 0,1}^{k} \leq\left|g_{p, 0,1}^{k}-g_{p, 0,0}^{k}\right| u_{p, 0,0}^{k+1}+g_{p, 0,1}^{k} u_{p, 0,1}^{k+1}+g_{p, 0,0}^{k} \frac{\alpha_{p, 0}^{k}}{w_{p, 0,0}^{k}} \leq B_{2} \Delta z+B_{1,1} B_{2}+\frac{c_{0} B_{1,1}}{\zeta_{2}} .
$$


Applying the preceding result and estimate (5.4.7) to (9.16) and enlarging $B_{3,8}$ as necessary we obtain the final result.

Proof of Lemma 5.5. From (4.1), the triangle inequality, and assumptions (B1), (B2), and (B8) and Lemma 5.4, for all $j=1, \ldots, N-1 l=1, \ldots, R-1$ we get

$$
\begin{aligned}
& \left(1-\lambda \Delta t+\frac{\Delta t}{\Delta x} g_{p, j, l}^{k}+\frac{\Delta t}{\Delta z} w_{p, j, l}^{k}\right)\left|u_{p, j+1, l}^{k+1}-u_{p, j, l}^{k+1}\right|-\frac{\Delta t}{\Delta x}\left|u_{p, j, l}^{k+1}-u_{p, j-1, l}^{k+1}\right| g_{p, j-1, l}^{k} \\
& -\frac{\Delta t}{\Delta z}\left|u_{p, j+1, l-1}^{k+1}-u_{p, j, l-1}^{k+1}\right| w_{p, j, l-1}^{k} \leq\left|u_{p, j+1, l}^{k}-u_{p, j, l}^{k}\right|+\frac{\Delta t}{2}\|\eta\|_{\infty} B_{2} B_{1} \Delta x+\frac{\Delta t}{2} B_{3} B_{1} \Delta x \\
& +\frac{\Delta t}{2}\|\eta\|_{\infty} B_{2} \sum_{s=1}^{j-1}\left|u_{p, j+1-s, l}^{k}-u_{p, j-s, l}^{k}\right| \Delta x+B_{3} \Delta x \Delta t+\Delta t B_{3} \sum_{i=1}^{n} u_{i, j+1, l}^{k} \Delta x \\
& +\Delta t\|Q\|_{\infty} \sum_{i=1}^{n}\left|u_{i, j+1, l}^{k}-u_{i, j, l}^{k}\right|+2 B_{3} u_{p, j}^{k+1}{ }^{k+1, l} \Delta x \Delta t+B_{3} u_{p, j, l}^{k+1} \Delta x \Delta t \\
& +n B_{3} u_{p, j, l}^{k+1} \Delta x \Delta t+\Delta t B_{1} B_{3} u_{p, j, l}^{k+1} \Delta x+\frac{\Delta t}{\Delta z} B_{3}\left|u_{p, j+1, l}^{k+1}-u_{p, j+1, l-1}^{k+1}\right| \Delta x
\end{aligned}
$$

Multiplying inequality (9.17) by $\Delta z$ and summing over $j=1, \ldots, N-1$ and $l=1, \ldots, R$

$$
\begin{aligned}
& \sum_{j=1}^{N-1} \sum_{l=1}^{R}\left(1-\lambda \Delta t+\frac{\Delta t}{\Delta x} g_{p, j, l}^{k}+\frac{\Delta t}{\Delta z} w_{p, j, l}^{k}\right)\left|u_{p, j+1, l}^{k+1}-u_{p, j, l}^{k+1}\right| \Delta z \\
& -\sum_{j=1}^{N-1} \sum_{l=1}^{R} \frac{\Delta t}{\Delta x}\left|u_{p, j, l}^{k+1}-u_{p, j-1, l}^{k+1}\right| g_{p, j-1, l}^{k} \Delta z-\sum_{j=1}^{N-1} \sum_{l=1}^{R} \frac{\Delta t}{\Delta z}\left|u_{p, j+1, l-1}^{k+1}-u_{p, j, l-1}^{k+1}\right| w_{p, j, l-1}^{k} \Delta z \\
& =(1-\lambda \Delta t) \sum_{j=1}^{N-1} \sum_{l=1}^{R}\left|u_{p, j+1, l}^{k+1}-u_{p, j, l}^{k+1}\right| \Delta z+\sum_{l=1}^{R} \frac{\Delta t}{\Delta x}\left|u_{p, N, l}^{k+1}-u_{p, N-1, l}^{k+1}\right| g_{p, N-1, l}^{k} \Delta z \\
& -\sum_{l=1}^{R} \frac{\Delta t}{\Delta x}\left|u_{p, 1, l}^{k+1}-u_{p, 0, l}^{k+1}\right| g_{p, 0, l}^{k} \Delta z+\sum_{j=1}^{N-1} \frac{\Delta t}{\Delta z}\left|u_{p, j+1, R}^{k+1}-u_{p, j, R}^{k+1}\right| w_{p, j, R}^{k} \Delta z-\sum_{j=1}^{N-1} \frac{\Delta t}{\Delta z} \mid u_{p, j+1,0}^{k+1} \\
& -u_{p, j, 0}^{k+1}\left|w_{p, j, 0}^{k} \Delta z \leq\left(1+B_{5,1} \Delta t\right) \sum_{j=1}^{N-1} \sum_{l=1}^{R}\right| u_{p, j+1, l}^{k}-u_{p, j, l}^{k} \mid \Delta z \\
& +\Delta t B_{5,1} \sum_{i=1}^{n} \sum_{j=1}^{N-1} \sum_{l=1}^{R}\left|u_{i, j+1, l}^{k}-u_{i, j, l}^{k}\right| \Delta z+\Delta t B_{5,1} \sum_{j=1}^{N-1} \sum_{l=1}^{R}\left|u_{p, j+1, l}^{k+1}-u_{p, j+1, l-1}^{k+1}\right| \Delta x
\end{aligned}
$$

where $B_{5,1} \geq \max \left\{0.5\|\eta\|_{\infty} B_{2} B_{1} x_{\max } z_{\max }+0.5 B_{3} B_{1} x_{\max } z_{\max }+B_{3} x_{\max } z_{\max }+(n+4) B_{3} B_{1}+\right.$ $\left.B_{1}^{2} B_{3}, 0.5\|\eta\|_{\infty} B_{2} x_{\max },\|Q\|_{\infty}, B_{3}\right\}$. By assumption (B1) and Lemma 5.4 .

$$
\begin{aligned}
& (1-\lambda \Delta t) \sum_{j=1}^{N-1} \sum_{l=1}^{R}\left|u_{p, j+1, l}^{k+1}-u_{p, j, l}^{k+1}\right| \Delta z \leq\left(1+B_{5,1} \Delta t\right) \sum_{j=1}^{N-1} \sum_{l=1}^{R}\left|u_{p, j+1, l}^{k}-u_{p, j, l}^{k}\right| \Delta z \\
& +\Delta t B_{5,1} \sum_{i=1}^{n} \sum_{j=1}^{N-1} \sum_{l=1}^{R}\left|u_{i, j+1, l}^{k}-u_{i, j, l}^{k}\right| \Delta z+\Delta t B_{5,1} \sum_{j=1}^{N-1} \sum_{l=1}^{R}\left|u_{p, j+1, l}^{k+1}-u_{p, j+1, l-1}^{k+1}\right| \Delta x \\
& +\sum_{j=1}^{N-1} \frac{\Delta t}{\Delta z}\left|u_{p, j}^{k+1}+u_{p, j, 0}^{k+1}\right| w_{p, j, 0}^{k} \Delta z+\sum_{l=1}^{k} \frac{\Delta t}{\Delta x}\left|u_{p, 1, l}^{k+1}-u_{p, 0, l}^{k+1}\right| g_{p, 0, l}^{k} \Delta z \\
\leq & \left(1+B_{5,1} \Delta t\right) \sum_{j=1}^{N-1} \sum_{l=1}^{R}\left|u_{p, j+1, l}^{k}-u_{p, j, l}^{k}\right| \Delta z+\Delta t B_{5,1} \sum_{i=1}^{n} \sum_{j=1}^{N-1} \sum_{l=1}^{R}\left|u_{i, j+1, l}^{k}-u_{i, j, l}^{k}\right| \Delta z \\
& +\Delta t B_{5,1} T V_{z}\left(u_{p}^{k+1}\right)+B_{3,8} \Delta t+\sum_{l=1}^{R} \frac{\Delta t}{\Delta x}\left|u_{p, 1, l}^{k+1}-u_{p, 0, l}^{k+1}\right| g_{p, 0, l}^{k} \Delta z .
\end{aligned}
$$

Combining the preceding result with estimate (5.4.8) from Lemma 5.4 we arrive at

$$
\begin{aligned}
\left(1-B_{5,1,1} \Delta t\right) T V_{x}\left(u_{p}^{k+1}\right) \leq & \left(1+B_{5,1} \Delta t\right) T V_{x}\left(u_{p}^{k}\right)+\Delta t\left(B_{5,1}+B_{3,8}\right) T V_{x}\left(\vec{u}^{k}\right) \\
& +\Delta t B_{5,1} T V_{z}\left(u_{p}^{k+1}\right)+B_{3,8}\left(2+T V_{z}\left(\vec{u}^{k}\right)\right) \Delta t,
\end{aligned}
$$

where $B_{5,1,1}=\max \left\{\lambda, B_{3,2}\right\}$. Similarly applying the triangle inequality to (4.1), assumptions (B1), (B2), and (B8) and Lemma 5.4 then summing over $j=1, \ldots, N$ and $l=1, \ldots, R-1$,

$$
\begin{aligned}
& \sum_{j=1}^{N} \sum_{l=1}^{R-1}\left(1-\lambda \Delta t+\frac{\Delta t}{\Delta x} g_{p, j, l}^{k}+\frac{\Delta t}{\Delta z} w_{p, j, l}^{k}\right)\left|u_{p, j, l+1}^{k+1}-u_{p, j, l}^{k+1}\right| \Delta x \\
& -\sum_{j=1}^{N} \sum_{l=1}^{R-1} \frac{\Delta t}{\Delta x}\left|u_{p, j-1, l+1}^{k+1}-u_{p, j-1, l}^{k+1}\right| g_{p, j-1, l}^{k} \Delta x-\sum_{j=1}^{N} \sum_{l=1}^{R-1} \frac{\Delta t}{\Delta z}\left|u_{p, j, l}^{k+1}-u_{p, j, l-1}^{k+1}\right| w_{p, j, l-1}^{k} \Delta x \\
\leq & \sum_{j=1}^{N} \sum_{l=1}^{R-1}\left|u_{p, j, l+1}^{k}-u_{p, j, l}^{k}\right| \Delta x+\frac{\Delta t}{2} B_{3} B_{2} B_{1} x_{\max } \\
& +\frac{\Delta t}{2}\|\eta\|_{\infty} B_{2} x_{\max } \sum_{l=1}^{R-1} \sum_{s=1}^{N}\left(\left|u_{p, s, l+1}^{k}-u_{p, s, l}^{k}\right|+\left|u_{p, s, l+1}^{k+1}-u_{p, s, l}^{k+1}\right|\right) \Delta x \\
& +B_{3} x_{\max } z_{\max } \Delta t+\Delta t B_{3} B_{1}+\Delta t|| Q \|_{\infty} \sum_{i=1}^{n} \sum_{j=1}^{N} \sum_{l=1}^{R-1}\left|u_{i, j, l+1}^{k}-u_{i, j, l}^{k}\right| \Delta x \\
& +\Delta t B_{3} B_{1}+\Delta t B_{3} \sum_{j=1}^{N} \sum_{l=1}^{R-1}\left|u_{p, j, l+1}^{k+1}-u_{p, j-1, l+1}^{k+1}\right| \Delta z \\
& +2 B_{1} \Delta t+n B_{3} B_{1} \Delta t+\Delta t B_{2}\left(B_{3} B_{1}+\|\eta\|_{\infty} x_{\max } \sum_{j=1}^{N} \sum_{l=1}^{R-1}\left|u_{p, j, l+1}^{k}-u_{p, j, l}^{k}\right| \Delta x\right)
\end{aligned}
$$


Simplifying and applying (B1) we have

$$
\begin{aligned}
& (1-\lambda \Delta t) \sum_{j=1}^{N} \sum_{l=1}^{R-1}\left|u_{p, j, l+1}^{k+1}-u_{p, j, l}^{k+1}\right| \Delta x \\
\leq & \left(1+\Delta t B_{5,2}\right) \sum_{j=1}^{N} \sum_{l=1}^{R-1}\left|u_{p, j, l+1}^{k}-u_{p, j, l}^{k}\right| \Delta x+\Delta t B_{5,2} \sum_{i=1}^{n} \sum_{j=1}^{N} \sum_{l=1}^{R-1}\left|u_{i, j, l+1}^{k}-u_{i, j, l}^{k}\right| \Delta x \\
& +\Delta t B_{5,2} \sum_{l=1}^{R-1} \sum_{j=1}^{N}\left|u_{p, j, l+1}^{k+1}-u_{p, j, l}^{k+1}\right| \Delta x+\Delta t B_{5,2} \sum_{j=1}^{N} \sum_{l=1}^{R-1}\left|u_{p, j, l+1}^{k+1}-u_{p, j-1, l+1}^{k+1}\right| \Delta z \\
& +\sum_{l=1}^{R-1} \frac{\Delta t}{\Delta x}\left|u_{p, 0, l+1}^{k+1}-u_{p, 0, l}^{k+1}\right| g_{p, 0, l}^{k} \Delta x+\sum_{j=1}^{N} \frac{\Delta t}{\Delta z}\left|u_{p, j, 1}^{k+1}-u_{p, j, 0}^{k+1}\right| w_{p, j, 0}^{k} \Delta x+B_{5,2} \Delta t
\end{aligned}
$$

where the last step follows from the fact that $B_{5,2} \geq 2 \max \left\{1.5 B_{3} B_{2} B_{1} x_{\max }+B_{3} x_{\max } z_{\max }+(n+\right.$ 2) $\left.B_{3} B_{1}+2 B_{1}, 1.5 B_{2}\|\eta\|_{\infty} x_{\max },\|Q\|_{\infty}, B_{3}\right\}$. Applying the previous Lemma 5.4 we arrive at

$$
\begin{aligned}
\left(1-B_{5,1,2} \Delta t\right) T V_{z}\left(u_{p}^{k+1}\right) \leq & \left(1+2 B_{5,2} \Delta t\right) T V_{z}\left(u_{p}^{k}\right)+\Delta t B_{5,2} T V_{x}\left(u_{p}^{k+1}\right) \\
& +\max \left\{B_{5,2}, B_{3,8}\right\}\left(1+T V_{z}\left(\vec{u}^{k+1}\right)\right) \Delta t+B_{5,2} \Delta t
\end{aligned}
$$

where $B_{5,1,2}=\max \left\{\lambda, B_{3,3}\right\}$. Combining (9.18) and (9.19),

$$
\begin{aligned}
& \left(1-C_{T V_{1}} \Delta t\right)\left(T V_{x}\left(u_{p}^{k+1}\right)+T V_{z}\left(u_{p}^{k+1}\right)\right) \\
\leq & \left(1+C_{T V_{2}} \Delta t\right)\left(T V_{x}\left(u_{p}^{k}\right)+T V_{z}\left(u_{p}^{k}\right)\right)+C_{T V_{3}}\left(T V_{x}\left(u_{p}^{k+1}\right)+T V_{z}\left(u_{p}^{k+1}\right)\right) \Delta t \\
& +C_{T V_{4}}\left(T V_{x}\left(\vec{u}^{k}\right)+T V_{z}\left(\vec{u}^{k}\right)\right) \Delta t+C_{T V_{5}}\left(T V_{z}\left(\vec{u}^{k+1}\right)\right) \Delta t+C_{T V_{6}} \Delta t,
\end{aligned}
$$

where $C_{T V_{1}}=\max \left\{B_{5,1,1}, B_{5,1,2}\right\}, C_{T V_{2}}=2 B_{5,2}+B_{5,1}, C_{T V_{3}}=B_{5,1}+B_{5,2}, C_{T V_{4}}=B_{3,8}+B_{5,1}$, $C_{T V_{5}}=B_{3,8}+B_{5,2}$ and $C_{T V_{6}}=3 B_{3,8}+2 B_{5,1}$. Summing over $p=1, \ldots, n$ we get

$$
\left(1-\Delta t C_{5}\right)\left(T V_{x}\left(\vec{u}^{k+1}\right)+T V_{z}\left(\vec{u}^{k+1}\right)\right) \leq\left(1+\Delta t C_{6}\right)\left(T V_{z}\left(\vec{u}^{k}\right)+T V_{x}\left(\vec{u}^{k}\right)\right)+C_{6} \Delta t .
$$

where $C_{5}=C_{T V_{1}}+C_{T V_{3}}+C_{T V_{5}}$ and $C_{6}=\max \left\{C_{T V_{2}}+C_{T V_{4}}, n C_{T V_{5}}, n C_{T V_{6}}\right\}$. The constant $B_{5}$ can be obtained from (9.20) via induction, elementary calculations, and assumption (B7). This completes the proof.

Proof of Lemma 5.6. Multiplying the first row in the numerical scheme 4.1 by $\Delta x \Delta z$ we obtain

$$
\begin{aligned}
\frac{u_{p, j, l}^{k+1}-u_{p, j, l}^{k}}{\Delta t} \Delta x \Delta z= & -\left(\left(g_{p, j, l}^{k}-g_{p, j-1, l}^{k}\right) u_{p, j-1, l}^{k+1}+\left(u_{p, j, l}^{k+1}-u_{p, j-1, l}^{k+1}\right) g_{p, j-1, l}^{k}\right) \Delta z \\
& -\left(\left(w_{p, j, l}^{k}-w_{p, j, l-1}^{k}\right) u_{p, j, l-1}^{k+1}+\left(u_{p, j, l}^{k+1}-u_{p, j, l-1}^{k+1}\right) w_{p, j, l-1}^{k}\right) \Delta x \\
& -\mu_{p, j, l}^{k} u_{p, j, l}^{k+1} \Delta x \Delta z-\sum_{i=1}^{k} Q_{i, p, j, l}^{k} u_{p, j, l}^{k+1} \Delta x \Delta z-u_{p, j, l}^{k+1} \sum_{m=1}^{N} \eta_{p, m, j, l}^{k} u_{p, m, l}^{k} \Delta x \Delta x \Delta z \\
& +\frac{1}{2} \sum_{s=1}^{j} \eta_{p, j-s, s, l}^{k} l_{p, j-s, l}^{k} u_{p, s, l}^{k+1} \Delta x \Delta x \Delta z+S_{p, j, l}^{B, k} \Delta x \Delta z+\sum_{i=1}^{n} Q_{p, i, j, l}^{k} u_{i, j, l}^{k} \Delta x \Delta z
\end{aligned}
$$

Applying the triangle inequality, summing over $p=1, \ldots, n, l=1, \ldots, R, j=1, \ldots, N$, and applying Lemma 5.4,

$$
\begin{aligned}
\sum_{p=1}^{n} \sum_{l=1}^{R} \sum_{j=1}^{N}\left|\frac{u_{p, j, l}^{k+1}-u_{p, j, l}^{k}}{\Delta t}\right| \Delta x \Delta z \leq & \left(2 B_{3}+B_{1,1}+1.5 c_{0} B_{2} x_{\text {max }}+2 n c_{0}\right) B_{1} \\
& +B_{1,1} B_{5}+B_{1,1} x_{\max } z_{\max } \leq \bar{L}
\end{aligned}
$$

and thus

$$
\sum_{p=1}^{n} \sum_{l=1}^{R} \sum_{j=1}^{N}\left|\frac{u_{p, j, l}^{r}-u_{p, j, l}^{q}}{\Delta t}\right| \Delta x \Delta z \leq \sum_{p=1}^{n} \sum_{l=1}^{R} \sum_{j=1}^{N} \sum_{k=q}^{r-1}\left|\frac{u_{p, j, l}^{k+1}-u_{p, j, l}^{k}}{\Delta t}\right| \Delta x \Delta z \leq B_{6}|r-q| .
$$

Similarly, using the estimates in (5.4.1) and (5.4.6) and similar arguments as above we obtain the Lipschitz condition for $Y_{p, \ell, l}^{k}$ in $z$ and $t$. 
Proof of Lemma 5.7. First define $v_{p, j, l}^{k}=u_{p, j, l}^{k}-\hat{u}_{p, j, l}^{k}$, for $l=1, \ldots, R, j=1, \ldots, N$ and $G_{l}^{k}=$ $\sum_{p=1}^{n}\left|\phi_{p, l}^{k}-\hat{\phi}_{p, l}^{k}\right|+\sum_{p=1}^{n} \sum_{\ell=1}^{m}\left|Y_{p, \ell, l}^{k}-\hat{Y}_{p, \ell, l}^{k}\right|$ for all $l=0, \ldots, R$, and observe that $v_{p, j, l}^{k}$ satisfies

$$
\begin{aligned}
& \left(1+g_{p, j, l}^{k} \frac{\Delta t}{\Delta x}+w_{p, j, l}^{k} \frac{\Delta t}{\Delta z}\right)\left|v_{p, j, l}^{k+1}\right|-\frac{\Delta t}{\Delta x} g_{p, j-1, l}^{k}\left|v_{p, j-1, l}^{k+1}\right|-\frac{\Delta t}{\Delta z} w_{p, j, l-1}^{k}\left|v_{p, j, l-1}^{k+1}\right| \\
& \leq \mid \\
& \quad\left|v_{p, j, l}^{k}\right|+\frac{\Delta t}{2}\left(c_{0} B_{2} \sum_{s=1}^{j-1}\left|v_{p, s, l}^{k+1}\right| \Delta x+B_{2} \sum_{s=1}^{j-1}\left(c_{0}\left|v_{p, j-s, l}^{k}\right|+L_{\eta} G_{l_{0}^{k}}^{k} B_{2}\right) \Delta x\right) \\
& \quad+\Delta t L_{S^{B}} G_{l_{0}^{k}}^{k}+\Delta t c_{0} \sum_{i=1}^{n}\left|v_{i, j, l}^{k}\right|+\Delta t L_{Q} G_{l_{0}^{k}}^{k} \sum_{i=1}^{n} \hat{u}_{i, j, l}^{k} \\
& \quad+\Delta t L_{g_{x}} G_{l_{0}^{k}}^{k} \hat{u}_{p, j, l}^{k+1}+\frac{\Delta t}{\Delta x} L_{g} G_{l_{0}^{k}}^{k}\left|\hat{u}_{p, j, l}^{k+1}-\hat{u}_{p, j-1, l}^{k+1}\right|+\Delta t L_{w_{z}} G_{l_{0}^{k}}^{k} \hat{u}_{p, j, l}^{k+1}+\frac{\Delta t}{\Delta z} L_{w} G_{l_{0}^{k}}^{k}\left|\hat{u}_{p, j, l}^{k+1}-\hat{u}_{p, j, l-1}^{k+1}\right| \\
& \quad+\Delta t L_{\mu} G_{l_{0}^{k}}^{k} \hat{u}_{p, j, l}^{k+1}+\Delta t L_{Q} G_{l_{0}^{k}}^{k} \sum_{i=1}^{n} \hat{u}_{p, j, l}^{k+1}+\Delta t B_{2} \sum_{q=1}^{N}\left(c_{0}\left|v_{p, q, l}^{k}\right|+L_{\eta} G_{l_{0}^{k}}^{k} B_{2}\right) \Delta x,
\end{aligned}
$$

where $l_{0}^{k}$ is the maximal index such that $G_{l_{0}^{k}}^{k}=\max _{l=0, \ldots, R}\left(\sum_{p=1}^{n}\left|\phi_{p, l}^{k}-\hat{\phi}_{p, l}^{k}\right|\right)+\max _{l=0, \ldots, R}\left(\sum_{p=1}^{n} \sum_{\ell=1}^{m}\left|Y_{p, \ell, l}^{k}-\hat{Y}_{p, \ell, l}^{k}\right|\right)$.

For ease of notation we hereinafter denote $G_{l_{0}^{k}}^{k}=G^{k}$. Multiplying by $\Delta x \Delta z$ and summing over $j=$ $1, \ldots, N, l=1, \ldots, R$ we have

$$
\begin{aligned}
& \sum_{l=1}^{R} \sum_{j=1}^{N}\left|v_{p, j, l}^{k+1}\right| \Delta x \Delta z+\frac{\Delta t}{\Delta x} \sum_{l=1}^{R}\left(g_{p, N, l}^{k}\left|v_{p, N, l}^{k+1}\right|-g_{p, 0, l}^{k}\left|v_{p, 0, l}^{k+1}\right|\right) \Delta x \Delta z \\
& \quad+\frac{\Delta t}{\Delta z} \sum_{j=1}^{N}\left(w_{p, j, R}^{k}\left|v_{p, j, R}^{k+1}\right|-w_{p, j, 0}^{k}\left|v_{p, j, 0}^{k+1}\right|\right) \Delta z \Delta x \\
& \leq \sum_{l=1}^{R} \sum_{j=1}^{N}\left|v_{p, j, l}^{k}\right| \Delta x \Delta z+\frac{\Delta t}{2} c_{0} B_{2} x_{\max } \sum_{l=1}^{R} \sum_{j=1}^{N}\left|v_{p, j, l}^{k+1}\right| \Delta x \Delta z \\
& \quad+\frac{\Delta t}{2} c_{0} B_{2} x_{\max } \sum_{l=1}^{R} \sum_{j=1}^{N}\left|v_{p, j, l}^{k}\right| \Delta x \Delta z+\frac{\Delta t}{2} B_{2}^{2} L_{\eta} G^{k} x_{\max }^{2} z_{\max } \\
& \quad+\Delta t L_{S^{B}} G^{k} x_{\max } z_{\max }+\Delta t c_{0} \sum_{i=1}^{n} \sum_{l=1}^{R} \sum_{j=1}^{N}\left|v_{i, j, l}^{k}\right| \Delta x \Delta z \\
& \quad+\Delta t L_{Q} G^{k} B_{1}+\Delta t L_{g_{x}} G^{k} B_{1}+\Delta t L_{g} G^{k} T V_{z}\left(\hat{u}_{p}^{k+1}\right) \\
& \quad+\Delta t L_{w_{z}} G^{k} B_{1}+\Delta t L_{w} G^{k} T V_{x}\left(\hat{u}_{p}^{k+1}\right)+\Delta t L_{\mu} G^{k} B_{1}+\Delta t L_{Q} G^{k} n B_{1} \\
& \quad+\Delta t c_{0} B_{2} x_{\max } \sum_{l=1}^{R} \sum_{j=1}^{N}\left|v_{p, j, l}^{k}\right| \Delta x \Delta z+\Delta t L_{\eta} G^{k} B_{2}^{2} x_{\max }^{2} z_{\max } \\
& \left.\quad+1+\Delta t C_{6,1}\right) \sum_{l=1}^{R} \sum_{j=1}^{N}\left|v_{p, j, l}^{k}\right| \Delta x \Delta z+\Delta t C_{6,1} \sum_{l=1}^{R} \sum_{j=1}^{N}\left|v_{p, j, l}^{k+1}\right| \Delta x \Delta z \\
& \quad+\Delta t C_{6,1} \sum_{i=1}^{n} \sum_{l=1}^{R} \sum_{j=1}^{N}\left|v_{i, j, l}^{k}\right| \Delta x \Delta z+\Delta t C_{6,2} G^{k} .
\end{aligned}
$$

where $C_{6,1}=\max \left\{c_{0}, 1.5 c_{0} B_{2} x_{\max }\right\}$ and $C_{6,2}=L_{S^{B}} x_{\max } z_{\max }+L_{Q} B_{1}+L_{g_{x}} B_{1}+L_{g} B_{5}+L_{w_{z}} B_{1}+$ $L_{w} B_{5}+L_{\mu} B_{1}+L_{Q} n B_{1}+1.5 B_{2}^{2} L_{\eta} x_{\max }^{2} z_{\max }$. Thus

$$
\begin{aligned}
& \sum_{l=1}^{R} \sum_{j=1}^{N}\left|v_{p, j, l}^{k+1}\right| \Delta x \Delta z \leq\left(1+\Delta t C_{6,1}\right) \sum_{l=1}^{R} \sum_{j=1}^{N}\left|v_{p, j, l}^{k}\right| \Delta x \Delta z+\Delta t C_{6,1} \sum_{l=1}^{R} \sum_{j=1}^{N}\left|v_{p, j, l}^{k+1}\right| \Delta x \Delta z \\
& \quad+\Delta t C_{6,1} \sum_{i=1}^{n} \sum_{l=1}^{R} \sum_{j=1}^{N}\left|v_{i, j, l}^{k}\right| \Delta x \Delta z+\Delta t \sum_{l=1}^{R} g_{p, 0, l}^{k}\left|v_{p, 0, l}^{k+1}\right| \Delta z+\Delta t \sum_{j=1}^{N} w_{p, j, 0}^{k}\left|v_{p, j, 0}^{k+1}\right| \Delta x \\
& \quad+\Delta t C_{6,2} G^{k} .
\end{aligned}
$$

Note also that

$$
\Delta t \sum_{l=1}^{R} g_{p, 0, l}^{k}\left|v_{p, 0, l}^{k+1}\right| \Delta z \leq\left(B_{2} L_{g} z_{\max }+L_{F} z_{\max }+B_{1} L_{\beta}\right) G^{k} \Delta t+c_{0}\left\|\vec{v}^{k}\right\|_{1} \Delta t
$$

and

$$
\Delta t \sum_{j=1}^{N} w_{p, j, 0}^{k}\left|v_{p, j, 0}^{k+1}\right| \Delta x \leq\left(B_{2} L_{w} G^{k}+L_{\alpha} \sum_{p=1}^{n}\left|\Phi_{p}^{k}-\hat{\Phi}_{p}^{k}\right|\right) x_{\max } \Delta t
$$

and combining the preceding expansions with (9.24) and summing over $p=1, \ldots, n$ we have

$$
\begin{aligned}
& \left\|\vec{v}^{k+1}\right\|_{1} \leq\left(1+\Delta t\left((n+1) C_{6,1}+n c_{0}\right)\right)\left\|\vec{v}^{k}\right\|_{1}+\Delta t C_{6,1}\left\|\vec{v}^{k+1}\right\|_{1} \\
& \quad+\Delta t\left(C_{6,2}+B_{2} L_{g} z_{\max }+L_{F} z_{\max }+B_{1} L_{\beta}+B_{2} L_{w} x_{\max }\right) G^{k}+L_{\alpha} x_{\max } \sum_{p=1}^{n}\left|\Phi_{p}^{k}-\hat{\Phi}_{p}^{k}\right| \Delta t \\
& \leq\left(1+\Delta t C_{6,3}\right)\left\|\vec{v}^{k}\right\|_{1}+\Delta t C_{6,1}\left\|\vec{v}^{k+1}\right\|_{1}+\Delta t C_{6,4} G^{k}+C_{6,5} \sum_{p=1}^{n}\left|\Phi_{p}^{k}-\hat{\Phi}_{p}^{k}\right| \Delta t .
\end{aligned}
$$


where $C_{6,3} \geq\left((n+1) C_{6,1}+n c_{0}\right), C_{6,4} \geq C_{6,2}+B_{2} L_{g} z_{\max }+L_{F} z_{\max }+B_{1} L_{\beta}+B_{2} L_{w} x_{\max }$ and $C_{6,5} \geq L_{\alpha} x_{\text {max }}$. Next observe that for all $l=0, \ldots, R$,

$$
\begin{aligned}
\sum_{p=1}^{n} \sum_{\ell=1}^{m}\left|Y_{p, \ell, l}^{k+1}-\hat{Y}_{p, \ell, l}^{k+1}\right| \leq \sum_{p=1}^{n} \sum_{\ell=1}^{m}\left(1+\sum_{i=1}^{n} \mathcal{Q}_{i, p, \ell, l}^{k} \Delta t+h_{p, \ell, l}^{k} \Delta t\right)\left|Y_{p, \ell, l}^{k+1}-\hat{Y}_{p, \ell, l}^{k+1}\right| \\
\leq \\
\quad\left(1+n c_{0} \Delta t\right) \sum_{p=1}^{n} \sum_{\ell=1}^{m}\left|Y_{p, \ell, l}^{k}-\hat{Y}_{p, \ell, l}^{k}\right| \\
\quad+2 n B_{2} \sum_{p=1}^{n} \sum_{\ell=1}^{m} L_{\mathcal{Q}}\left(\left|\sigma_{p, \ell, l}^{k}-\hat{\sigma}_{p, \ell, l}^{k}\right|+\left|Y_{p, \ell, l}^{k}-\hat{Y}_{p, \ell, l}^{k}\right|\right) \Delta t \\
\quad+\sum_{p=1}^{n} \sum_{\ell=1}^{m} L_{f}\left(\left|\sigma_{p, \ell, l}^{k}-\hat{\sigma}_{p, \ell, l}^{k}\right|+\left|Y_{p, \ell, l}^{k}-\hat{Y}_{p, \ell, l}^{k}\right|\right) \Delta t \\
\quad+B_{2} \sum_{p=1}^{n} \sum_{\ell=1}^{m} L_{h}\left(\left|\sigma_{p, \ell, l}^{k}-\hat{\sigma}_{p, \ell, l}^{k}\right|+\left|Y_{p, \ell, l}^{k}-\hat{Y}_{p, \ell, l}^{k}\right|\right) \Delta t \\
\leq \\
\leq\left(1+C_{6,6} \Delta t\right) \sum_{p=1}^{n} \sum_{\ell=1}^{m}\left|Y_{p, \ell, l}^{k}-\hat{Y}_{p, \ell, l}^{k}\right|+C_{6,7} \sum_{p=1}^{n} \sum_{\ell=1}^{m}\left|\sigma_{p, \ell, l}^{k}-\hat{\sigma}_{p, \ell, l}^{k}\right| \Delta t .
\end{aligned}
$$

where the constants satisfy $C_{6,6} \geq n c_{0}+2 n L_{\mathcal{Q}} B_{2}+L_{f}+B_{2} L_{h}$ and $C_{6,7} \geq 2 n L_{\mathcal{Q}} B_{2}+L_{f}+B_{2} L_{h}$. Combining (9.25) and (9.26) we have

$$
\begin{aligned}
& \left(1-\Delta t C_{6,1}\right)\left(\left\|\vec{v}^{k+1}\right\|_{1}+\max _{l=0,1, \ldots, R} \sum_{p=1}^{n} \sum_{\ell=1}^{m}\left|Y_{p, \ell, l}^{k+1}-\hat{Y}_{p, \ell, l}^{k+1}\right|\right) \leq \\
& \left(1+\Delta t C_{6,8}\right)\left(\left\|\vec{v}^{k}\right\|_{1}+\max _{l=0,1, \ldots, R} \sum_{p=1}^{n} \sum_{\ell=1}^{m}\left|Y_{p, \ell, l}^{k}-\hat{Y}_{p, \ell, l}^{k}\right|\right) \\
& \quad+\Delta t C_{6,4} G^{k}+C_{6,5} \sum_{p=1}^{n}\left|\Phi_{p}^{k}-\hat{\Phi}_{p}^{k}\right| \Delta t+C_{6,7} \max _{l=0,1, \ldots, R} \sum_{p=1}^{n} \sum_{\ell=1}^{m}\left|\sigma_{p, \ell, l}^{k}-\hat{\sigma}_{p, \ell, l}^{k}\right| \Delta t .
\end{aligned}
$$

where $C_{6,8}=\max \left\{C_{6,3}, C_{6,6}\right\}$. By the triangle inequality and (B6), we obtain $\sum_{p=1}^{n}\left|\Phi_{p}^{k}-\hat{\Phi}_{p}^{k}\right| \leq c_{0}\left\|\vec{v}^{k}\right\|_{1}$, $G^{k} \leq \max _{l=0,1, \ldots, R} \sum_{p=1}^{n} \sum_{\ell=1}^{m}\left|Y_{p, \ell, l}^{k}-\hat{Y}_{p, \ell, l}^{k}\right|+c_{0}\left\|\vec{v}^{k}\right\|_{1}$ and that $\max _{l=0,1, \ldots, R} \sum_{p=1}^{n} \sum_{\ell=1}^{m}\left|\sigma_{p, \ell, l}^{k}-\hat{\sigma}_{p, \ell, l}^{k}\right| \leq m c_{0}\left\|\vec{v}^{k}\right\|_{1}$. As a result, there exists a constant $C^{\dagger}$ such that

$$
\begin{aligned}
& \left\|\vec{v}^{k+1}\right\|_{1}+\max _{l=0,1, \ldots, R} \sum_{p=1}^{n} \sum_{\ell=1}^{m}\left|Y_{p, \ell, l}^{k+1}-\hat{Y}_{p, \ell, l}^{k+1}\right| \\
& \leq\left(\frac{1+C^{\dagger} \Delta t}{1-C_{6,1} \Delta t}\right)\left(\left\|\vec{v}^{k}\right\|_{1}+\max _{l=0, \ldots, R} \sum_{p=1}^{n} \sum_{\ell=1}^{m}\left|Y_{p, \ell, l}^{k}-\hat{Y}_{p, \ell, l}^{k}\right|\right) .
\end{aligned}
$$

This completes the proof. 